ARTICLE

Received 22 Feb 2016 | Accepted 10 Aug 2016 | Published 30 Sep 2016

DOI: $10.1038 /$ ncomms12864

OPEN

\title{
An endoplasmic reticulum stress-regulated IncRNA hosting a microRNA megacluster induces early features of diabetic nephropathy
}

Mitsuo Kato ${ }^{1}$, Mei Wang ${ }^{1}$, Zhuo Chen ${ }^{1}$, Kirti Bhatt ${ }^{1}$, Hyung Jung Oh¹, Linda Lanting ${ }^{1}$, Supriya Deshpande ${ }^{1}$ Ye Jia', Jennifer Y.C. Lai ${ }^{2}$, Christopher L. O'Connor ${ }^{2}$, YiFan Wu², Jeffrey B. Hodgin ${ }^{3}$, Robert G. Nelson ${ }^{4}$, Markus Bitzer ${ }^{2}$ \& Rama Natarajan ${ }^{1}$

It is important to find better treatments for diabetic nephropathy (DN), a debilitating renal complication. Targeting early features of $\mathrm{DN}$, including renal extracellular matrix accumulation (ECM) and glomerular hypertrophy, can prevent disease progression. Here we show that a megacluster of nearly 40 microRNAs and their host long non-coding RNA transcript (Inc-MGC) are coordinately increased in the glomeruli of mouse models of $\mathrm{DN}$, and mesangial cells treated with transforming growth factor- $\beta 1$ (TGF- $\beta 1$ ) or high glucose. Lnc-MGC is regulated by an endoplasmic reticulum (ER) stress-related transcription factor, CHOP. Cluster microRNAs and Inc-MGC are decreased in diabetic Chop ${ }^{-1-}$ mice that showed protection from DN. Target genes of megacluster microRNAs have functions related to protein synthesis and ER stress. A chemically modified oligonucleotide targeting Inc-MGC inhibits cluster microRNAs, glomerular ECM and hypertrophy in diabetic mice. Relevance to human DN is also demonstrated. These results demonstrate the translational implications of targeting Inc-MGC for controlling DN progression.

\footnotetext{
${ }^{1}$ Department of Diabetes Complications and Metabolism, Diabetes Metabolism Research Institute, Beckman Research Institute of City of Hope, Duarte, California 91010, USA. ${ }^{2}$ Department of Internal Medicine, University of Michigan, Ann Arbor, Michigan 48109, USA. ${ }^{3}$ Department of Pathology, University of Michigan, Ann Arbor, Michigan 48109, USA. ${ }^{4}$ Diabetes Epidemiology and Clinical Research Section, National Institute of Diabetes and Digestive and Kidney Diseases, National Institutes of Health, Phoenix, Arizona 85014, USA. Correspondence and requests for materials should be addressed to M.K. (email: mkato@coh.org) or to R.N. (email: rnatarajan@coh.org).
} 
$\mathrm{D}$ iabetic nephropathy (DN) is one of the most common and debilitating complications of diabetes for which better therapies are sorely needed. The 'early' features of DN include glomerular mesangial expansion and hypertrophy, increased renal accumulation of extracellular matrix (ECM) proteins such as collagens and fibronectin, as well as podocyte effacement ${ }^{1,2}$. The expression of profibrotic transforming growth factor- $\beta 1$ (TGF- $\beta 1$ ) is increased in mesangial cells (MCs) and other renal cells in diabetes and mediates many of these adverse effects in DN. Factors associated with the pathogenesis of DN such as angiotensin II and high glucose (HG) increase TGF- $\beta 1$ expression in MCs in vitro and in vivo ${ }^{3-7}$. Signals from the activated TGF- $\beta 1$ receptor complex are transduced to the nucleus by Smad proteins, which regulate TGF- $\beta 1$-induced gene expression $^{8}$. However, it is not fully clear how diabetic conditions and TGF- $\beta 1$ regulate the genes that increase the hypertrophy, protein synthesis and fibrosis associated with DN.

MicroRNAs (miRNAs) are endogenously produced, short non-coding RNAs ( $\sim 20-22$ nucleotides) that play key roles in post-transcriptional regulation of gene expression. miRNAs silence genes by repressing their translation or inducing the degradation of target mRNAs ${ }^{9}$. Since nearly $60 \%$ of mRNAs in the genome are targeted by miRNAs, they regulate many cellular functions and pathophysiological states including kidney diseases $^{6,9,10}$. Several miRNAs have been implicated in the pathogenesis of $\mathrm{DN}$ and also as possible biomarkers or therapeutic targets ${ }^{2,6,10}$. Antisense inhibitors have been widely used to inhibit specific miRNAs $s^{11-13}$ and some clinical trials are ongoing $^{14,15}$. Inhibition of key miRNAs by chemically modified antisense oligonucleotides or by genetic knockout (KO) attenuated early manifestations of DN in mice (ECM accumulation and glomerular hypertrophy) and albuminuria (a later feature of $\mathrm{DN})^{2,6,10-12,16-21}$. Therefore, targeting specific miRNAs that mediate the early features of DN could be an effective approach to prevent progression of DN. In this study, we observed that an unique megacluster of nearly 40 miRNAs were coordinately increased along with their host transcript (a long non-coding RNA (lncRNA)) in the diabetic kidney and mediated several features of DN. Furthermore, inhibition of the host transcript decreased the expression of the cluster miRNAs, and attenuated early features of DN in vitro in MCs, and in vivo in mice.

\section{Results}

Cluster miRNAs and host RNA are increased in diabetic kidney. RNA samples from the glomeruli of diabetic (streptozotocin (STZ) injected) and control mice were profiled by small RNA (miRNA)-sequencing and analysed by our methods ${ }^{22}$. In the diabetic mice glomeruli, we found a significant increase in the expression of numerous miRNAs that are among the megacluster of miRNAs within the DLK-DIO3 genomic region (mouse chr12, human chr14) ${ }^{23}$, while miR-822 located outside of this cluster was unaltered (Fig. 1a-c and Supplementary Fig. 1). Gene Set Enrichment analyses (GSEA) ${ }^{24}$ indicated that these cluster miRNAs were significantly enriched among the miRNAs increased in the diabetic mice (Fig. 1d). PCR validation revealed significant increase in the levels of 40 of these cluster miRNAs in the glomeruli of both STZ injected and $\mathrm{db} / \mathrm{db}$ mice (models of types 1 and 2 diabetes, respectively) compared with their corresponding controls (Fig. 2a). Genome organization shows that the megacluster (MGC) is hosted by a lncRNA, termed 'Inc-MGC', 3' region of which overlaps with Mirg and middle region with Gm2922, other ncRNAs (Fig. 1c and Supplementary Fig. 1). miR-379 is located at the $5^{\prime}$ end, miR-494 and miR-495 in the middle, and miR-377 downstream (Fig. 1c). The $5^{\prime}$ region of
lnc-MGC was cloned by $5^{\prime}$ rapid amplification of complementary DNA (cDNA) ends (RACE; Fig. 2b). This lnc-MGC host transcript was also increased in the glomeruli of diabetic mice (Fig. 2c). TGF- $\beta 1$ and HG significantly increased the expression of miR-379, miR-494, miR-495 and miR-377 as well as lnc-MGC, but not miR-882, compared with serum depleted (SD) or normal glucose (NG) controls respectively in cultured mouse MC (MMC; Fig. 2d).

Since copy numbers of lncRNAs are in general relatively low (for example, HOTTIP copy number is 0.3 per cell) ${ }^{25}$, we calculated the copy numbers of the $5^{\prime}$ region of lnc-MGC cloned by us. Lnc-MGC was expressed at 3-4 copies per cell in control MMC, and 4-6 copies per cell in HG or TGF- $\beta$ treated MMC (Supplementary Fig. 2), suggesting adequate expression of lnc-MGC for further study. Although miR-377and miR-382 have been implicated in renal fibrosis and HG response ${ }^{26,27}$, our data show that multiple miRNAs of the megacluster and host lncRNA are concomitantly increased in the glomeruli and MCs under diabetic conditions. Levels of these cluster miRNAs as well as lnc-MGC were also higher in the renal cortex and glomeruli of diabetic mice with more advanced diabetic kidney disease including significant proteinuria (22 weeks after onset of diabetes) $^{17}$ (Supplementary Fig. 3a). These results suggest that the cluster miRNAs are co-regulated with the host lnc-MGC and may play key roles in the pathogenesis of DN. To date only few lncRNAs have been reported as hosts for some miRNAs under diabetic conditions, for e.g., RP23-298H6.1-001 hosting miR-216a and miR-217 (refs 6,11,28-30).

Targets of the miRNA cluster. miRNA target prediction algorithms indicated that miRNAs in the megacluster have several common target genes. Therefore, the $3^{\prime}$ UTRs of at least 25 genes are similarly targeted by $8-13$ cluster miRNAs, with some genes having more than two miRNA target sites (Supplementary Fig. 4a). These targets include RNA binding proteins and translational regulators including CUGBP2 (ref. 31), PUM2 (ref. 32), TNRC6B (ref. 33), CPEB4 (ref. 34), HuR (ref. 35), transcription factors and co-factors (Arid2, BHC80 (ref. 36), NF1a/b), ATF3, Endoplasmic Reticulum (ER) Degradation Enhancer, Mannosidase Alpha-Like $3(E D E M 3)^{37}$ and tumour suppressor phosphatase and tensin homologue $(P T E N)^{38}$. In silico Ingenuity Pathway Analyses (IPA) suggested that these target gene sets have properties related to various molecular and cellular functions, human diseases, TGF- $\beta$ signalling and other pathways, renal development/function and renal toxicity (Supplementary Figs $4 \mathrm{~b}-\mathrm{f}$ and $5 \mathrm{a}-\mathrm{e})$. Furthermore, IPA network analyses also suggested strong activation of Akt kinase (as well as Akt regulators phosphatidyl inositol-3kinase and PTEN) and Erk kinase (Supplementary Fig. 6a,b), known regulators of cellular hypertrophy and ECM accumulation. Some of the identified target genes also regulate protein translation, protein synthesis, mRNA stability and miRNA processing ${ }^{39}$. Interestingly, BHC80 interacts with histone deactylases and Ets to promote chromatin condensation ${ }^{28,36}$. ATF3 is a repressor of C/EBP homologous protein (CHOP), which is involved in ER stress $s^{40-42}$ and a direct target of miR-494 (ref. 43), a miRNA in the miR-379 cluster that we confirmed to be increased in diabetic mice glomeruli. The binding sites of multiple cluster miRNAs in the $3^{\prime}$ UTRs of seven target genes (human and mouse) relevant to DN, namely EDEM3, ATF3, TNRC6B, CUGBP2, CPEB4 and PHF21A are shown in Supplementary Figs 7-20 (obtained from TargetScan and microRNA.org). Therefore, the genes targeted by megacluster miRNAs have several functions related to $\mathrm{DN}$, protein synthesis, ER stress, RNA binding and protein translation (Supplementary Figs 4-6). 
a

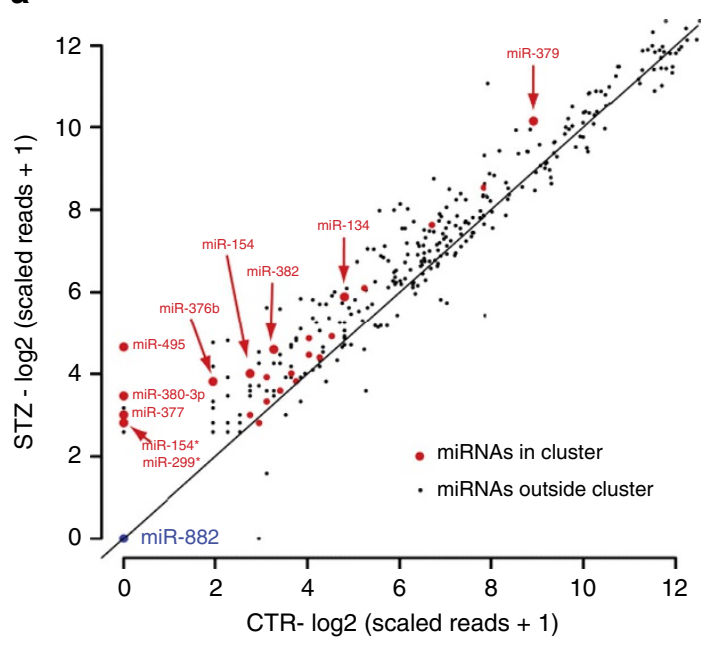

b

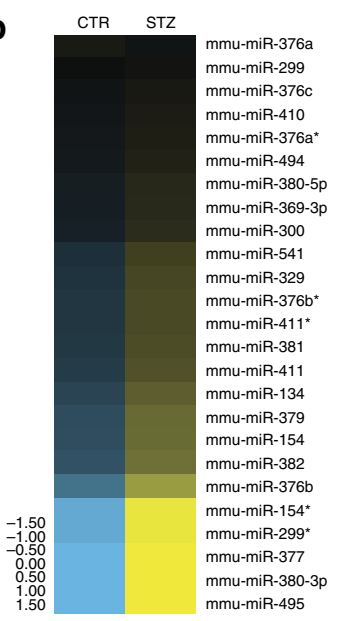

C

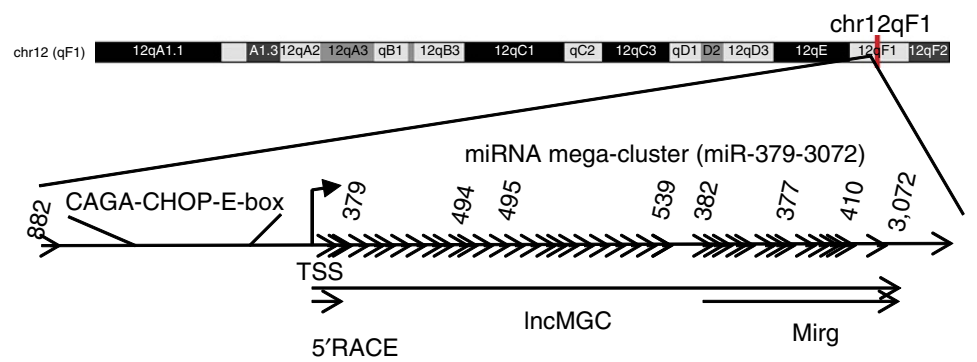

d

GSEA pre-rank analysis

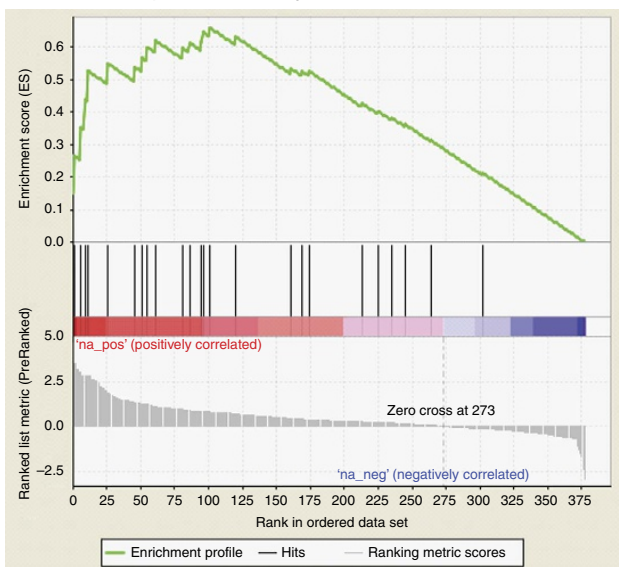

Enrichment score $=1.56(P=0.004)$.

Figure 1 | The miR-379 miRNA megacluster is increased in the glomeruli of diabetic mice. Small RNA (smRNA) sequencing was performed as previously described $^{22}$. (a) Scatter plot of miRNAs in kidney glomeruli from control (CTR, vehicle injected) and diabetic mice (STZ injected; 4 weeks post diabetes). The expression of each detectable miRNA in the form of log scaled reads was plotted with $x$ axis for CTR and $y$ axis for STZ. Each dot represents one miRNA. miRNAs in the miR-379 cluster were presented in red. Among these, the miRNAs upregulated by fold change $\geq 2$ are highlighted with bigger size dots and labelled with the corresponding miRNA names. miR-882 was plotted in blue as a negative control (outside the miR-379 cluster). (b) Heatmap of the miR-379 cluster miRNAs in CTR and STZ samples. The expression of each detectable miRNA within the miR-379 cluster in the two samples (CTR and STZ) were ordered by log2 fold change from low to high, mean-centered and shown in the heatmap. Blue represents lower than average expression in the two samples and yellow presents higher than average expression level. The expression of the detected miRNAs in this cluster was higher in STZ than CTR. (c) Genome structure of the mouse miR-379 megacluster region. This cluster is located within the largest miRNA cluster currently identified in the genome. It maps within the DLK-DIO3 genomic region (mouse chr 12, human chr14), which is home to several miRNAs and IncRNAs. TSS, transcription start site. miR-882 is located far-upstream of the miR-379 cluster and not covered by Inc-MGC. (d) Gene Set Enrichment Analysis (GSEA). All the miRNAs detected by smRNA-seq with at least 5 scaled reads in at least one sample are ranked by log2 fold change between STZ and CTR samples to generate ranked list and all the detectable miRNAs in the cluster are considered as a gene set. Pre-ranked gene set analysis (GSEA) applied on the gene set using the ranked list of all the miRNAs revealed that miRNAs in the miR-379 cluster were significantly enriched within the miRNAs upregulated in the STZ diabetic mice, with normalized enrichment score of $1.56(P=0.004)$. 

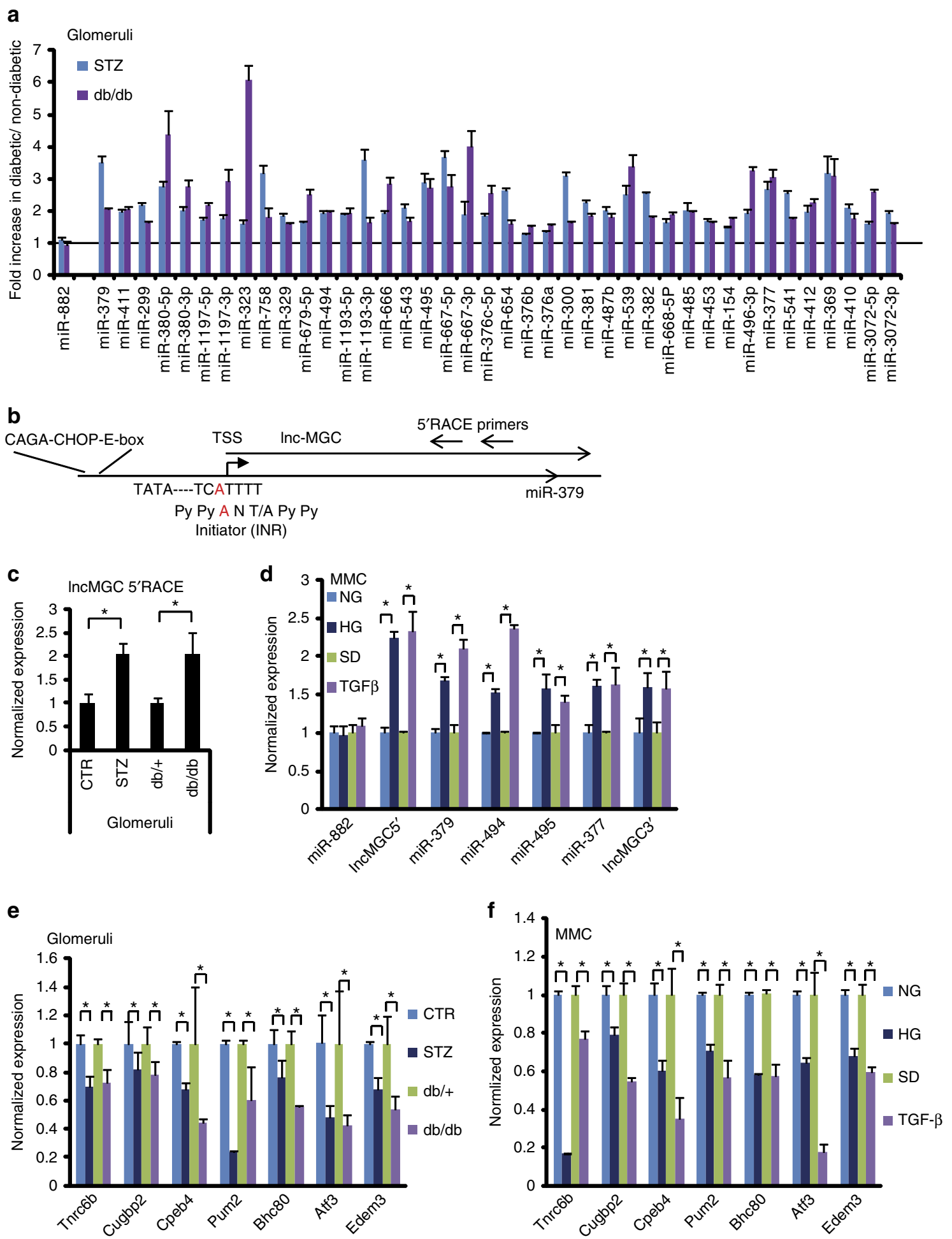

Figure 2 | Lnc-MGC and cluster miRNAs are upregulated under diabetic conditions. (a) Primers for almost all ( $\sim 40)$ miRNAs in miR-379 cluster were designed and the expression of 40 miRNAs were confirmed by RT-PCRs and found to be increased significantly, Benjamini-Hochberg adjusted $P<0.05$ (ref. 69) in glomeruli from diabetic mice (STZ, STZ-injected type 1 model, blue bars; db/db, type 2 diabetic model, red bars) compared with respective non-diabetic mice (vehicle injected or $\mathrm{db} /+$ ). Five mice in each group. Results are mean + s.e. from triplicate PCRs. No significant change was detected in the expression of miR-882 (outside of the miR-379 cluster). (b) 5'RACE of the region upstream of miR-379 identified the typical consensus sequence of initiator (INR; PyPyANT/APyPy) at the transcription start site. Smad-binding elements (CAGA repeats) and CHOP binding consensus and E-box sequences were found upstream of miR-379. (c) The expression of Inc-MGC in glomeruli from diabetic mice (STZ and db/db) compared with corresponding controls (vehicle injected CTR and $\mathrm{db} /+$ ). Five mice in each group. ${ }^{*} \mathrm{P}<0.05$, (d) The expression of Inc-MGC and cluster miRNAs in MMC treated with $25 \mathrm{mM} H \mathrm{HG}$ $(72 \mathrm{~h})$ or $10 \mathrm{ng} \mathrm{ml}^{-1}$ TGF- $\beta 1(24 \mathrm{~h})$ compared with respective controls normal glucose $(5.5 \mathrm{mM})$ or SD. respectively. (e) Significant lower expression of potential targets of miR-379 cluster in kidney glomeruli from diabetic mice (type $2 \mathrm{db} / \mathrm{db}$ and type $1 \mathrm{STZ}$ ) than the respective non-diabetic control mice. Five mice in each group. ${ }^{\star} P<0.05$. (f) Decrease of potential targets of miR-379 cluster in MMC treated with TGF- $\beta 1$ or HG. For experiments with MMC, results are mean + s.e. in triplicate PCRs from three independent culture experiments. ${ }^{\star} P<0.05$. 
The mRNA levels of Tnrc6b, Cugbp2, Cpeb4, Pum2, Bhc80, Atf 3 and Edem 3 were significantly reduced in the glomeruli from diabetic mice in vivo (db/db and STZ) compared with respective control mice (Fig. 2e), and in MMC treated with TGF- $\beta 1$ or HG in vitro (Fig. $2 \mathrm{f}$ ) compared with $\mathrm{SD}$ or normal glucose controls respectively. These targets have the potential to mediate the downstream effects of diabetes in a synergistic manner to augment renal hypertrophy and fibrosis in DN.

Upstream region of the miRNA megacluster and host lnc-MGC. Apart from the region upstream of lnc-MGC, no other clear putative promoter was evident throughout the miRNA cluster, suggesting lnc-MGC is a long transcript which serves as host for the cluster miRNAs. To identify a putative promoter that drives the expression of this genomic region, we carried out $5^{\prime}$ RACE experiments using primers spanning the miR-379 upstream region (Fig. 2b). This region has promoter-like features with TATA box and initiator (INR) consensus sequences ${ }^{44}$. CAGA repeats (Smad-binding elements) were found $\sim 3 \mathrm{~kb}$ upstream of miR-379 (Fig. 3a), suggesting regulation by TGF- $\beta 1$. Chromatin immunoprecipitation (ChIP) assays showed a significant increase in Smad2/3 occupancy at the miR-379 promoter Smad-binding sites (arrows, Fig. 3a) in MMCs treated with TGF- $\beta 1$ compared with SD control (Supplementary Fig. 21a). Smad2/3 occupancy peaked at $1 \mathrm{~h}$, and returned to normal by $24 \mathrm{~h}$, suggesting the miR-379 cluster is regulated by TGF- $\beta 1$ through rapid enrichment of $S \operatorname{mad} 2 / 3$ at the upstream region. We also identified binding sites for $\mathrm{CHOP}$, a transcription factor associated with the ER stress response, and an overlapping E-box $\sim 1.6 \mathrm{~kb}$ upstream of miR-379 (Fig. 3a), suggesting that the identified promoter of the lncRNA-MGC-miRNA cluster may be regulated by $\mathrm{CHOP}$.

Regulatory role of CHOP. CHOP protein was induced in MMCs treated with TGF- $\beta 1$, HG or osmotic control mannitol (Fig. 3b), suggesting that diabetic conditions and cellular stress upregulate CHOP, which increases transcription of lnc-MGC and the miRNA cluster. ChIP assays showed significant increase in $\mathrm{CHOP}$ occupancy at the CHOP binding sites in the miR-379 promoter in MMCs treated with HG or TGF- $\beta 1$ (Fig. 3c,d). The upstream $(-1.6 \mathrm{~kb})$ region with wild-type (WT) $\mathrm{CHOP}$, or the same region with mutated CHOP binding site (MT) was cloned into the pGL4 luciferase reporter and these plasmids transfected into MMC (Fig. 3e). The WT plasmid showed significantly higher luciferase activity than empty pGL4 whereas CHOP-MT showed significantly reduced promoter activity (Fig. 3e). Together with ChIP data, the CHOP binding site $(-1.6 \mathrm{~kb})$ is essential for the expression of lnc-MGC and miR-379 cluster. ChIP of E-box regulators (USF1, Tfe 3 and Zeb1) showed increased enrichment of E-box activators (USF1 and Tfe3), but decreased enrichment of
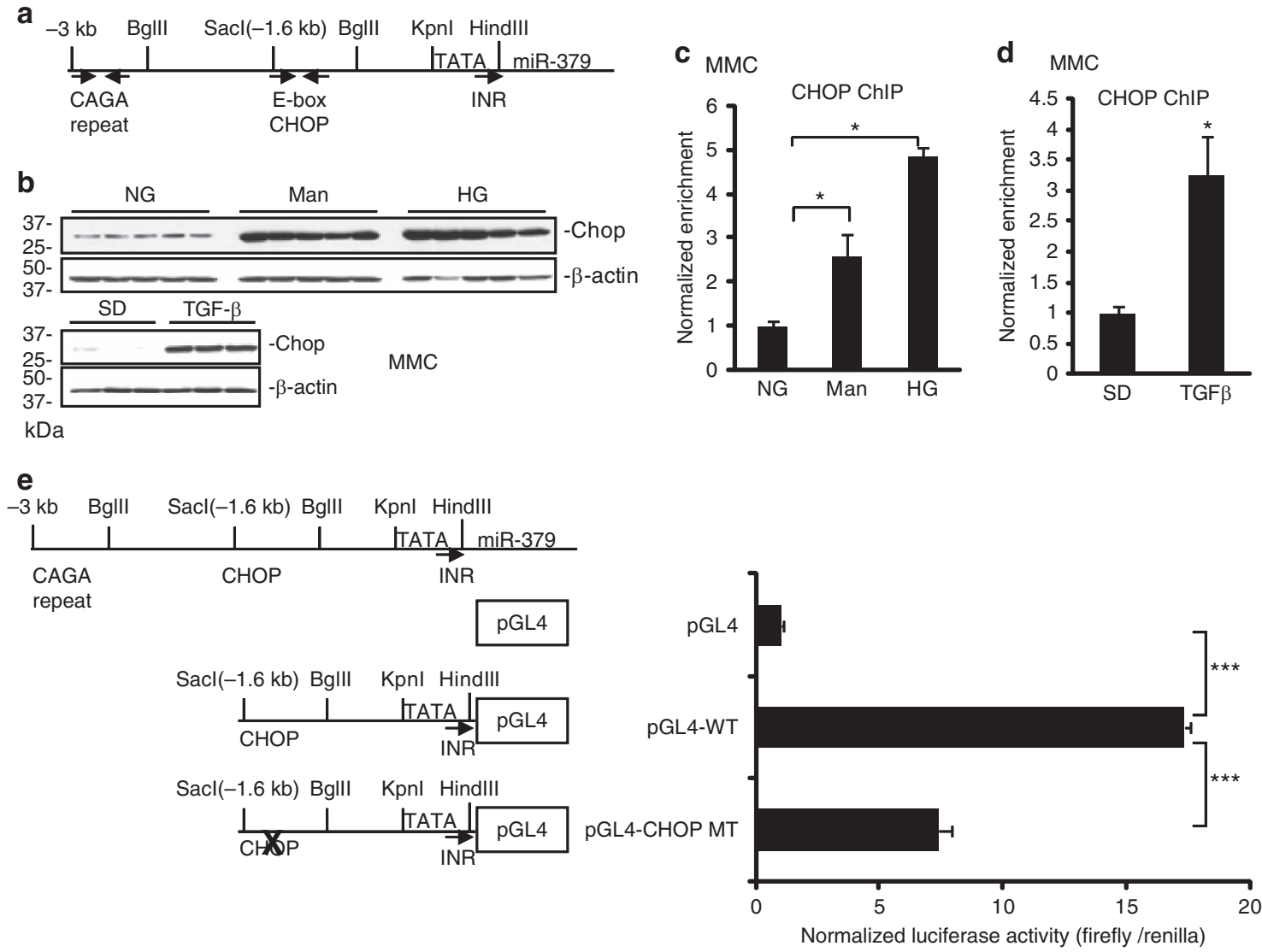

Figure 3 | Regulatory role of CHOP. (a) Genomic structure of the region upstream of miR-379. CAGA repeats (potential Smad-binding elements) were identified at $\sim 3 \mathrm{~kb}$ upstream of miR-379 and CHOP binding element and E-boxes were found close to a Sacl restriction enzyme site at $\sim 1.6 \mathrm{~kb}$ upstream of miR-379. (b) Chop protein levels were increased in MMC treated with TGF- $\beta 1$, HG or mannitol compared with respective controls. Wider (uncropped) scans are shown in Supplementary Fig. 38. (c,d) ChIP assays and ChIP-real-time PCRs. CHOP occupancy was enriched at the CHOP binding site in MMC treated with HG or mannitol (c) or TGF- $\beta 1$ (d). Results are mean + s.e. in triplicate PCRs from three independent ChIP experiments. ${ }^{\star} P<0.05$.

(e) The upstream $(-1.6 \mathrm{~kb}$ ) region, or the same region with mutated CHOP binding site, was cloned into the luciferase reporter pGL4 and plasmids were transfected into MMC. The WT plasmid showed significantly higher luciferase activity than empty pGL4 and mutation of CHOP site (MT) significantly reduced promoter activity. Results are mean + s.e. from triplicate reads of four independent cultures. ${ }^{\star \star \star} P<0.001$. 
the E-box repressor, Zeb1, in MMC treated with TGF- $\beta 1$ (Supplementary Fig. 21b-d). The E-box binding region might contribute to the regulation of the miR-379 cluster by mechanisms (miRNA-mediated increase of E-box activators and decrease of E-box repressor) similar to those identified in the regulation of collagens in MC under diabetic conditions ${ }^{16,45}$.

Figure $4 \mathrm{a}$ shows the efficacy of Chop short interfering RNA (siRNA). Chop siRNA significantly attenuated HG- and TGF- $\beta 1$-induced expression of miR-379 and lnc-MGC in MMC (Fig. 4b,c). Mir-495 and -377 as well as Mirg levels were also inhibited (Supplementary Fig. 21f-i). These results demonstrate that $\mathrm{CHOP}$ is a key transcriptional regulator of the miRNA cluster as well as its host lncRNA-MGC. Chop siRNA treatment significantly reversed the downregulation of a miRNA target (Tnrc6b) by HG and TGF- $\beta 1$ in MMC (Supplementary Fig. 22a). Chop siRNA also ameliorated TGF- $\beta 1$-induced expression of profibrotic genes Col1 $\alpha 2$, TGF- $\beta 1$, Coll $\alpha 4$ and Pai-1 (Supplementary Fig. 22b-g).

Cluster miRNAs and Inc-MGC in Chop-KO mice. Since our data implicated $\mathrm{CHOP}$ as one of the major regulators of lnc-MGC, we examined its in vivo functional role using Chop-KO
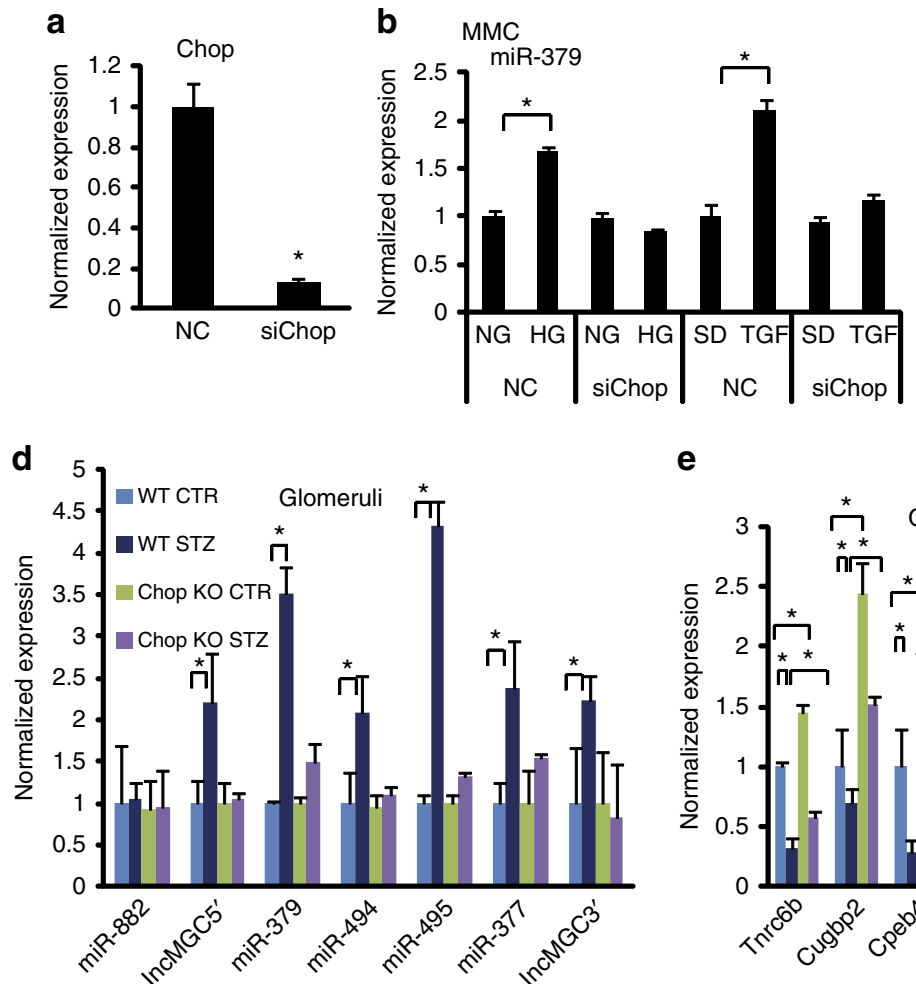

e

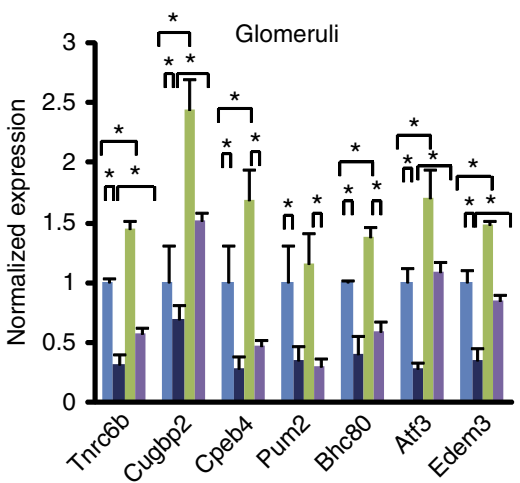

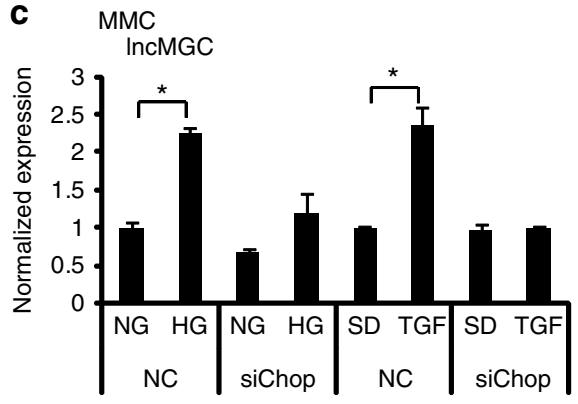

f g

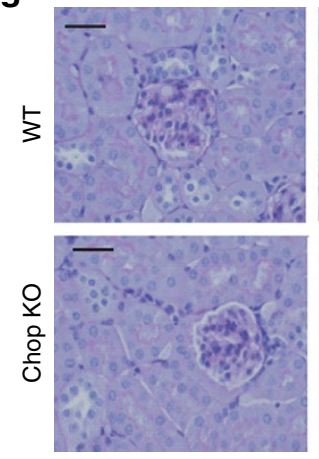

CTR

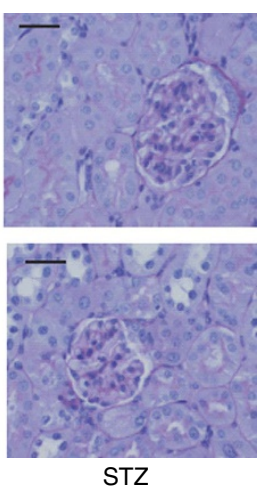

h

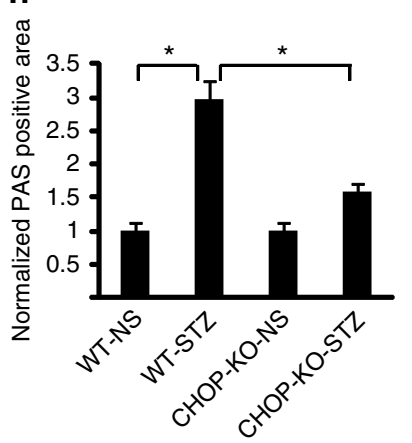

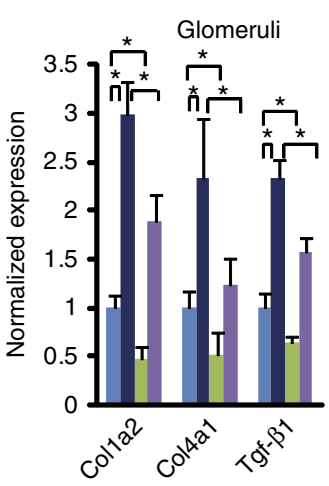

Figure 4 | Regulatory role of CHOP (using siRNA and Chop-KO mice). (a) Effects of Chop siRNA. Chop expression was significantly decreased by Chop siRNA in MMC. (b) Effects of Chop siRNA on the expression of miR-379. Chop siRNA inhibited TGF- $\beta 1$ or HG mediated increase of miR-379 in MMC but had no effect on basal levels of miR-379. (c) Effects of Chop siRNA on the expression of Inc-MGC. Chop siRNA inhibited the induction of Inc-MGC in MMC treated with TGF- $\beta 1$ or HG but had no effect on basal levels. Results are mean + s.e. in triplicate PCRs from three independent culture experiments, ${ }^{\star} P<0.05$. (d) The miR-379 cluster miRNAs are not induced in the kidneys of diabetic Chop-KO mice. The increases of Inc-MGC, miR-379, miR-494, miR-495 and miR-377 noted in glomeruli from diabetic WT mice were not observed in glomeruli from diabetic Chop-KO mice (five mice in each group). (e) Decrease of potential targets of miR-379 cluster in glomeruli from diabetic WT mice and restoration of their expression in Chop-KO mice. (f) Increase of profibrotic gene expression in glomeruli from diabetic WT mice was attenuated in Chop-KO mice. (g-i) Glomerular hypertrophy and ECM accumulation were increased in WT diabetic mice and these were attenuated in diabetic Chop-KO mice. Scale bar, $20 \mu \mathrm{m}$. (h) PAS staining in glomeruli from WT and Chop-KO mice. PAS positive area (ECM accumulation). (i) glomerular area (hypertrophy). ${ }^{\star} P<0.05$. 
mice. Diabetes was induced in Chop-KO and control WT mice with STZ injections. Chop-KO mice developed diabetes at the same rates as WT. Mice were euthanized four weeks after diabetes induction. Interestingly, diabetes-induced increases in candidate cluster miRNAs as well as lnc-MGC, which were evident in the glomeruli of diabetic WT mice, were abrogated in the glomeruli of diabetic Chop-KO mice (Fig. 4d). The levels of key targets of the miR-379 cluster were significantly lower in diabetic mice. However, their levels were significantly higher in Chop-KO mice than WT (Fig. 4e). The increased expression of profibrotic genes Coll $\alpha 2$, Col4 $\alpha 1$ and $T g f-\beta 1$ were attenuated in glomeruli from diabetic Chop-KO mice compared to diabetic WT mice (Fig. 4f). Histological analysis showed that Periodic acid-Schiff (PAS) staining and glomerular hypertrophy were clearly reduced in diabetic Chop-KO mice compared to diabetic WT (Fig. 4g-i).

We cultured MMCs from non-diabetic WT and Chop-KO mice and treated them with or without TGF- $\beta 1$. Both basal and TGF- $\beta 1$-induced increases in three cluster miRNAs and lnc-MGC (but not miR-882) were ameliorated in MMC derived from Chop-KO mice compared to WT mice (Supplementary Fig. 23a). In addition, the expression of candidate target genes of the miR-379 cluster was significantly higher in MMC from Chop-KO mice as compared to WT (basal and after TGF- $\beta 1$ treatment) (Supplementary Fig. 23b). Furthermore, both basal and TGF- $\beta 1$-induced expression of fibrotic genes, Collagen type I alpha2 (Coll $\alpha 2$ ), Col4 $\alpha 1$ and Tgf- $\beta 1$ (relevant to DN), were significantly attenuated in MMC from the Chop-KO mice compared to WT (Supplementary Fig. 23c). TGF- $\beta 1$-induced increase in cellular hypertrophy was ameliorated in MMCs from Chop-KO compared to WT mice (Supplementary Fig. 23d). Similar to the effects seen with TGF- $\beta 1$, HG-induced increases in lnc-MGC, cluster miRNAs and profibrotic genes, as well as decrease of target genes, were attenuated in MMC from Chop-KO mice (Supplementary Fig. 24a-c). When Chop-KO MMC were transfected with miR-379 mimic, significant increase of miR-379, profibrotic genes but decrease of targets were observed (Supplementary Fig. 24d-i), confirming that ectopic expression of decreased miRNAs in Chop-KO MMC reverses the expression of targets and profibrotic genes.

Role of ER stress in cluster miRNAs and lnc-MGC expression. To verify the role of ER stress, MMC were treated with tunicamycin (TM), a known ER stress inducer, and the expression of miR-379 cluster miRNAs examined. To optimize conditions for TM treatment, the expression of HSPA5 (heat shock $70 \mathrm{kDa}$ protein 5), also known as GRP78 (glucose-regulated protein, $78 \mathrm{kDa}$ ), was examined (Supplementary Fig. 25a). $50 \mathrm{ng} \mathrm{ml}^{-1}$ was the minimum dose of TM required to induce significant expression of HSPA5 as well as CHOP (Supplementary Fig. 25a-c). TM $\left(\sim 50 \mathrm{ng} \mathrm{ml}^{-1}\right)$ also upregulated lnc-MGC and key cluster miRNAs in MMC (Supplementary Fig. 25d-i). TM reciprocally decreased several targets of the miR-379 cluster including Edem3, a target of miR-379 (Supplementary Fig. 26a-g). Interestingly, a faster-migrating isoform of Edem3 protein was detected in TM-treated cells but not in MMC treated with TGF- $\beta 1$ (Supplementary Fig. 26g,h). This is reasonable because TM is an inhibitor of N-glycosylation and the fastermigrating form is an un-glycosylated form of Edem 3 that leads to loss of its activity to protect the cells from ER stress ${ }^{37}$. On the other hand, TGF- $\beta 1$ treatment decreased Edem 3 expression likely through induction of miR-379 since miR-379 mimic oligonucleotides (oligos) reduced Edem3 levels (Supplementary Fig. 26h,i). This illustrates two independent modes of Edem3 regulation, loss of $\mathrm{N}$-glycosylation and decrease of expression through miR-379 induction (Supplementary Fig. 26g-i). In parallel, profibrotic genes, such as Col1 $\alpha 2$, Col4 $\alpha 1$, fibronectin (FN) and Tgf- $\beta 1$ were also upregulated by TM (Supplementary Fig. 27a-d). These results suggest that TM (ER stress) increases lnc-MGC and the miR-379 cluster miRNAs, and enhances ER stress and DN phenotypes (hypertrophy and fibrosis) by inhibiting the miR-379 cluster targets.

Increased ER stress has been reported in animal models of $\mathrm{DN}^{46-51}$. XBP1 is spliced and activated by ER stress ${ }^{52}$. We observed increased Xbp1 splicing in MMC treated with TM, HG or TGF- $\beta 1$ (Supplementary Fig. 27e-g), suggesting that these factors induce ER stress. In diabetic glomeruli and MMC treated with TGF- $\beta 1$ or HG, although ATF4 or ATF6 were unaltered, we observed a significant decrease in Atf3 (Fig. 2e,f), a repressor of $\mathrm{CHOP}^{40-42}$ and also a direct target of miR-494 (ref. 43), a miR-379 cluster miRNA confirmed to be increased in diabetic glomeruli. Xbp1 splicing and Atf3 downregulation by miR-494 co-operate to upregulate Chop in MMC in response to HG or TGF- $\beta$.

Edem 3 is as a target of miR-379. Edem 3 protein levels were decreased in MMC treated with TGF- $\beta 1$, or in MMC transfected with miR-379 mimics (Supplementary Fig. 26h,i). A reporter plasmid encompassing the full $3^{\prime} \mathrm{UTR}$ of mouse Edem 3 gene (Full) was cotransfected into MMC along with miR-379 mimics (Supplementary Fig. 28a,b). TGF- $\beta 1$ treatment reduced the luciferase activity of this reporter compared to SD control. miR-379 mimics also inhibited the luciferase activity of this reporter compared to negative control mimics (CTR), suggesting Edem3 is a direct target of miR-379. Interestingly, a target site of $\mathrm{miR}-200 \mathrm{~b} / \mathrm{c}$ was also found in the $3^{\prime} \mathrm{UTR}$ of Edem 3 and miR-200b mimic oligos inhibited the reporter activity (Supplementary Fig. 28a,b). Because miR-200b/c are upregulated in glomeruli from diabetic mice and in MMC treated with TGF- $\beta 1^{2,6,16}$, miR-200 family and miR-379 cluster may collaborate to inhibit Edem 3 expression. miR-379 and miR-200b mimics had no effect on reporters containing partial deletion of the region harbouring miR-379 and miR-200b/c target sites in the $3^{\prime}$ UTR of Edem3, verifying these sites to be bonafide targets of miR-379 and $\mathrm{miR}-200 \mathrm{~b} / \mathrm{c}$ (Supplementary Fig. 28c). These results also suggest that one of the mechanisms by which the miR-379 cluster upregulated in diabetes contributes to $\mathrm{DN}$ is through ER stress promoted by loss of Edem3.

Inhibition of lnc-MGC by GapmeRs in vitro and in vivo. siRNAs designed to target lnc-MGC regions located upstream of miR-379 were effective to silence lnc-MGC and cluster miRNAs (Supplementary Fig. 29a,b). However, since this approach needed transfection with three siRNAs and not practical for in vivo targeting, we evaluated 'GapmeRs' to inhibit lnc-MGC expression.

Locked nucleic acid (LNA)-modified anti-miRNAs are useful to target specific miRNAs to protect diabetic mice from various features of $\mathrm{DN}^{12}$. LNA modification has several advantages, including reduced toxicity, lower dosing and efficient targeting. LNA GapmeRs are antisense oligos optimized for specific inhibition of RNA in vitro and in vivo ${ }^{53,54}$. GapmeRs induce RNase $\mathrm{H}$-dependent RNA degradation in the nucleus, and hence nuclear retained RNAs are effectively targeted. To knockdown lnc-MGC, four LNA-modified GapmeR oligos (MGC1, MGC5, MGC8 and MGC10) were designed (Supplementary Fig. 30a) having three LNAs at both 5' and 3' ends and ten DNAs in the centre, and a fully phosphorothioated backbone (Supplementary Fig. 30b). MMC were transfected with these GapmeRs or a control LNA GapmeR and the expression of lnc-MGC examined. The control LNA GapmeR oligo had the same modification as our lncRNA targeting GapmeR but with no homology to any 
known mRNA, miRNA, or lncRNA in mouse, rat and human. MGC10 consistently inhibited lnc-MGC expression significantly at $48 \mathrm{~h}$ after transfection in two independent experiments. Of the other three GapmeRs, MGC1 and MGC5 showed some inhibition, but were less effective than MGC10 and not consistent in their actions (Supplementary Fig. 30c,d). MGC10 also reduced the expression of lnc-MGC under TGF- $\beta 1$ treated conditions in MMC (Supplementary Fig. 30e). In addition, key miRNAs in the miR-379 megacluster were downregulated by MGC10 (Supplementary Fig. 31a-c). Furthermore, targets (Edem3, Tnrc6b and Bhc80) of the miR-379 cluster were upregulated by MGC10 (Supplementary Fig. 31d-g).

Because MGC10 was effective in reducing the expression of lnc-MGC and key miR-379 cluster miRNAs in MMC in vitro, we next tested its actions in mouse kidneys in vivo (Supplementary Fig. 32). Subcutaneous injection of $5 \mathrm{mg} \mathrm{kg}^{-1}$ MGC10 into normal mice significantly reduced the expression of lnc-MGC in their kidneys (by $24 \sim 72 \mathrm{~h}$ ) (Supplementary Fig. 32a,b). miR$379,-495$ and -377 (but not miR-882), were also reduced in the same samples (Supplementary Fig. 32c-f).

To confirm the renal delivery of MGC10, a fluorescent antisense LNA-modified probe was designed for in situ hybridization. Interestingly, by $24 \mathrm{~h}$ there was widespread signal in cytosolic regions of both glomerular and tubular regions in kidneys of mice injected with MGC10, while clear nuclear accumulation of MGC10 was observed in these kidney compartments at $48-72 \mathrm{~h}$ after the injection (Fig. 5). Only weak background signal was detected in the kidneys of vehicle (PBS) injected mice. A recent report suggested that phosphorothioated oligonucleotides are transported into the nucleus by a protein (TCP1) complex ${ }^{55}$. Because MGC10 is fully phosphorothioated, it is efficiently transported into the nucleus where it cleaves lnc-MGC RNA and thereby suppresses the expression of the miR-379 cluster (Supplementary Fig. 33).

Next, MGC10 was tested in diabetic mice. Five non-diabetic mice (NS), five un-injected diabetic mice (STZ), six diabetic mice injected with negative control GapmeR oligonucleotides $(\mathrm{STZ}+\mathrm{C})$ and six diabetic mice injected with $5 \mathrm{mg} \mathrm{kg}^{-1}$

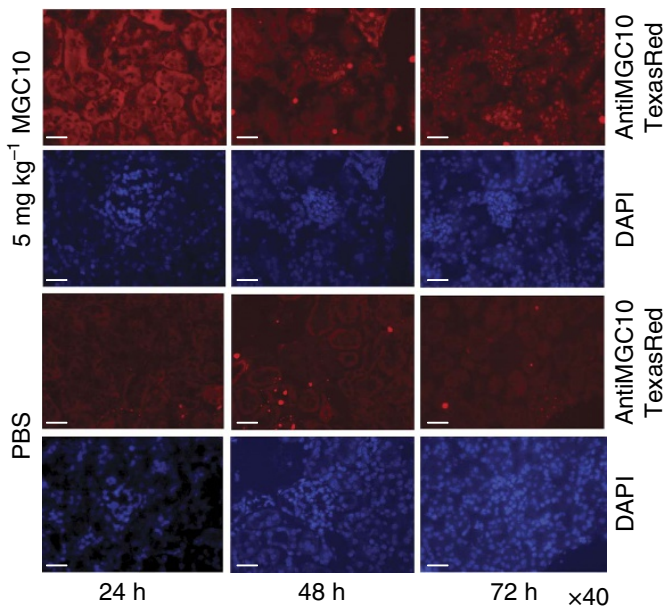

Figure 5 | Detection of Gapmer MGC10 in mouse kidney by in situ hybridization. $24 \mathrm{~h}$ after subcutaneous injection $\left(5 \mathrm{mg} \mathrm{kg}^{-1}\right)$ of $\mathrm{MGC10}$, red fluorescence signals (by in situ hybridization of renal sections with a Texas Red conjugated complementary probe) were detected in tubular and glomerular areas (mostly in cytoplasm). However, after $48 \mathrm{~h}$, nuclear

accumulation of MGC10 was observed and the signals were more enriched in nuclei at $72 \mathrm{~h}$ after injection. Blue 4,6-diamidino-2-phenylindole signals represent nuclear regions. Weak background was detected in the kidneys of mice injected with vehicle (PBS) as control. Scale bar, $20 \mu \mathrm{m}$.
MGC10 targeting lnc-MGC (STZ + MGC10) were examined at 5 weeks after the onset of diabetes (Supplementary Fig. 34a). Serum profiling showed significant increase in glucose levels in all diabetic mice (STZ, STZ + C and STZ + MGC10) compared to non-diabetic mice (NS). There was no significant difference in parameters of liver and kidney toxicity or inflammation (inflammatory cytokines) due to MGC10 injection, suggesting that MGC10 does not alter glucose levels, and does not induce significant toxicity or inflammation (Supplementary Figs 34b and $35 a, b)$.

Lnc-MGC expression was higher in the kidneys (cortex and glomeruli) from diabetic versus non-diabetic mice. Its expression was significantly reduced in kidneys of diabetic mice injected with MGC10 (STZ-MGC10) compared to control GapmeR (STZ-C), demonstrating that MGC10 is effective even in diabetic mice (Fig. 6a and Supplementary Fig. 36a-c). Expression of the cluster miRNAs, (miR-379, -494, -495, -377) but not miR-882, and Mirg depicted a similar pattern as lnc-MGC, demonstrating specificity to this cluster (Fig. 6a-f). Key targets (Edem3, Atf3, Tnrc6B, Cpeb4, Pum2) of the miR-379 cluster were reduced in kidneys from diabetic mice but their expression was restored in the kidney cortex and glomeruli of diabetic mice injected with MGC10 (Fig. 6g,h and Supplementary Fig. 36d-f). Profibrotic genes, Tgf- $\beta 1$, Colla2, Col4a1, Ctgf, which were upregulated in the kidneys of diabetic mice, were reduced in diabetic mice injected with MGC10 (Fig. 6i-l).

PAS staining of renal sections showed mesangial expansion and increased glomerular size in diabetic mice compared to that in non-diabetic mice and these features were reduced in diabetic mice injected with MGC10 (Fig. 7a-c). With respect to ER stress, immunostaining of Edem3, a target of miR-379, showed significant decrease in glomeruli of diabetic mice and this was reversed in glomeruli of diabetic mice injected with MGC10 (Fig. 7d,e).

TGF- $\beta$ or diabetic conditions (as well as ER stress) induce glomerular podocyte dysfunction and death ${ }^{56}$. In order to determine whether MGC10 confers any protection on podocytes in diabetes, we assessed podocyte effacement and glomerular basement membrane (GBM) thickness using electron microscopy (EM; Fig. 7f,g). Clear protection from diabetesinduced podocyte effacement and GBM thickening was observed in diabetic mice treated with the MGC10 compared to control oligo. Cell death measured by Tunel assay was increased in glomeruli of diabetic mice compared to non-diabetic mice, which was attenuated by MGC10 (Fig. 7h,i). These results suggest that MGC10 is effective in reducing the expression of not only lnc-MGC and miR-379 cluster miRNAs in vivo in diabetic mice, but also restores the expression of the cluster miRNA targets, inhibits profibrotic genes and prevents glomerular fibrosis, podocyte death, and hypertrophy in diabetic mice.

Human homologue of lnc-MGC, and inhibition by GapmeR. To test if the miR-379 cluster is similarly regulated in human cells, human MC (HMC) were treated with TGF- $\beta 1$ or HG. The human homologue of lnc-MGC (hlnc-MGC) was examined by PCR using human specific primers (Supplementary Fig. 37a). hlnc-MGC and miR-379 cluster miRNAs were increased by TGF- $\beta 1$ or HG in HMC, with miR-882 showing no significant difference (Fig. 8a,b). Since the target sequence in human hlnc-MGC has two-base mismatches, we designed a human version of GapmeR MGC10 (HMGC10) based on the human sequence (Supplementary Fig. 37a,b). Basic GapmeR chemistry was similar to the mouse MGC10. Transfection conditions in HMC were optimized for efficacy and cell viability (Supplementary Fig. 37c). hlnc-MGC levels were significantly reduced by HMGC10 in HMC compared to negative control 
a

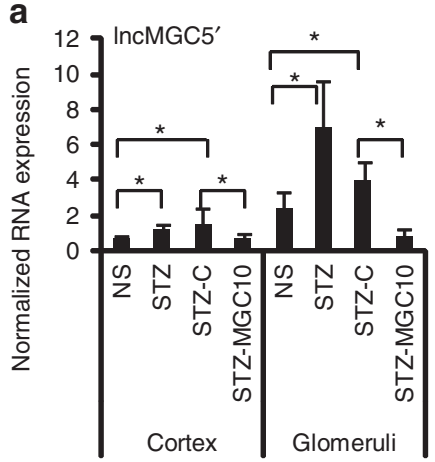

e

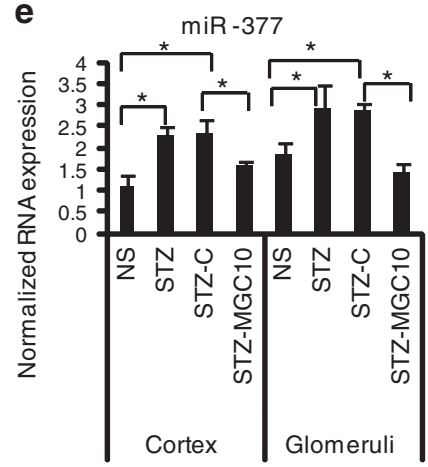

i

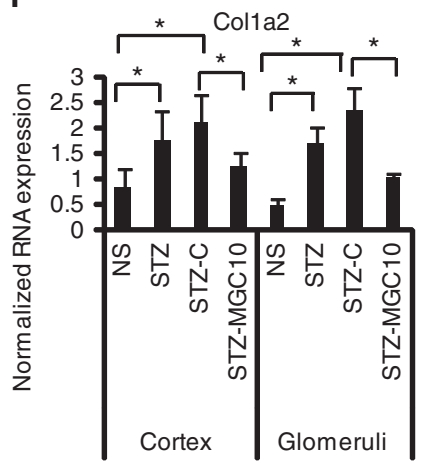

b

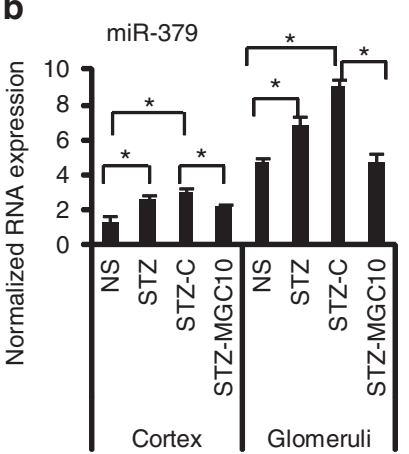

f

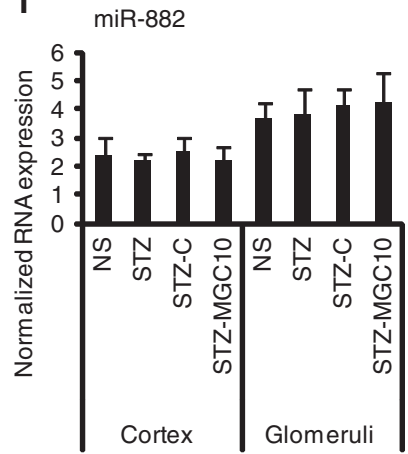

c

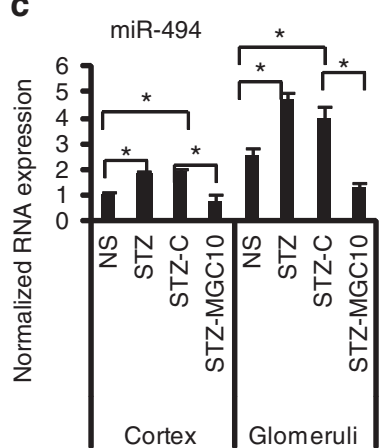

g

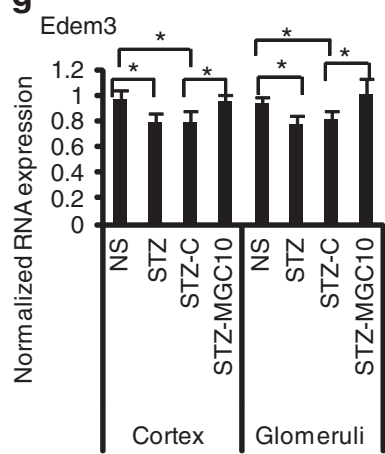

d

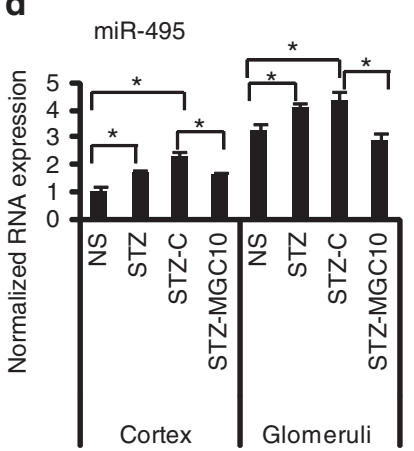

h

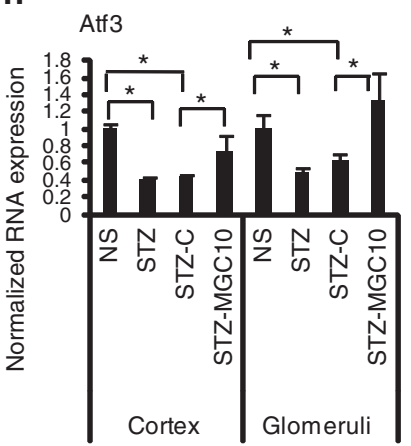

j

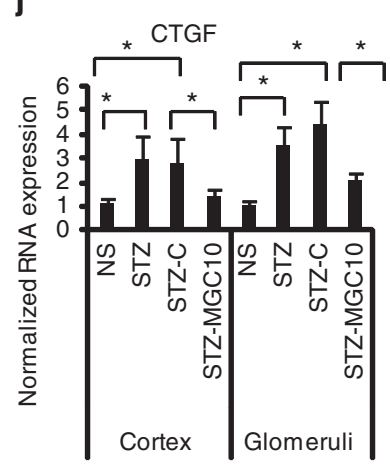

k

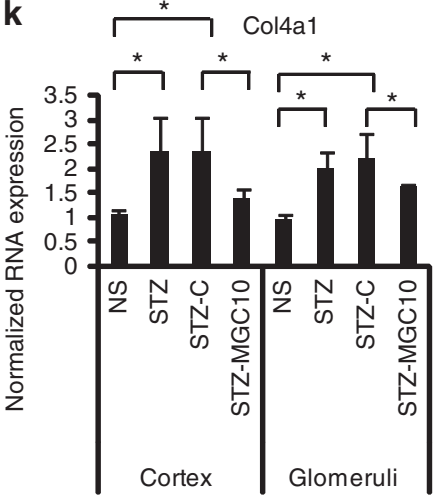

I

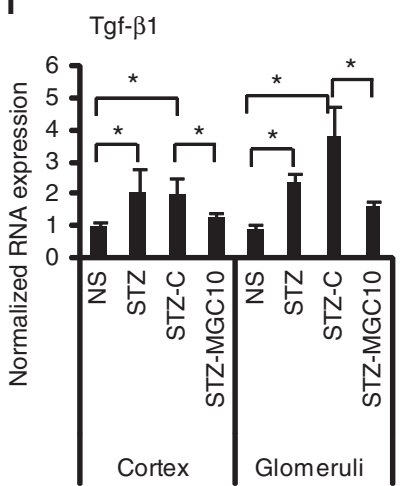

Figure 6 | In vivo effects of GapmeR targeting Inc-MGC (MGC10) in mice. (a) Increase of Inc-MGC in kidney cortex and glomeruli from STZ diabetic mice and its significant inhibition by MGC10 injection. Five mice for NS and STZ groups and six mice for STZ + C and STZ-MGC10 groups. Results are mean + s.e. in triplicate PCRs from each mouse. ${ }^{\star} P<0.05$. (b-f) Increase of miR-379 (b), miR-494 (c), miR-495 (d), miR-377 (e) but not miR-822 (f) in kidney cortex and glomeruli from STZ diabetic mice and their significant inhibition by injection of MGC10. (g,h) Decrease of miR-379 target Edem3 and miR-494 target Atf3 in kidney cortex and glomeruli from STZ diabetic mice and significant restoration of their levels by injection with MGC10. $(\mathbf{i}-\mathbf{I})$ Increase of profibrotic genes Col1a2 (i), Ctgf (j), Col4a1 (k) and Tgf- $\beta 1$ (I) in kidney cortex and glomeruli from STZ diabetic mice and significant inhibition by injection of MGC10. ${ }^{\star} P<0.05$.

(Supplementary Fig. 37d). Key targets, EDEM3 and CPEB4, were also decreased by TGF- $\beta 1$ in HMC (Supplementary Fig. 37e-g).

Human lnc-MGC expression was significantly inhibited by HMGC10 under both basal and TGF- $\beta 1$ treated conditions (Fig. 8a). Similar trends were observed for miR-379, miR-494, miR-495 and miR-377, but not miR-822, suggesting that inhibition of hlnc-MGC (host RNA) by HMGC10 reduces the cluster miRNAs in HMC treated with TGF- $\beta 1$ (Fig. 8a). Similarly, HG-induced expression of hlnc-MGC, and cluster miRNAs in HMC were significantly inhibited by HMGC10 (Fig. 8b). The reduction of candidate targets (EDEM3, ATF3, CPEB4 and CUGBP2) of the cluster miRNAs by TGF- $\beta 1$ was reversed by HMGC10 in HMC (Fig. 8c). Induction of profibrotic genes (TGF- $\beta 1, C O L 1 A 2, C O L 4 A 1, F N 1$ and CTGF) by TGF- $\beta 1$ was attenuated by HMGC10 (Fig. 8d). HG-induced reduction of target genes (EDEM3, ATF3, CPEB4 and CUGBP2) was reversed by HMGC10 (Fig. 8e). HG-induced increased expression of profibrotic genes (TGF- $\beta 1, C O L 1 A 2, C O L 4 A 1, F N 1$ and CTGF) was also ameliorated by HMGC10 in HMC (Fig. 8f). These results suggest that reduction of hlnc-MGC by HMGC10 can suppress the miR-379 cluster miRNAs, restore the expression of their targets and inhibit the profibrotic genes in HMC treated with TGF- $\beta 1$ or $\mathrm{HG}$, highlighting the potential translational significance.

Expression of miR-379 cluster miRNAs in human kidney tissue. We further examined if these cluster miRNAs are expressed in glomeruli of patients with diabetic kidney disease ${ }^{17}$. Several cluster miRNAs were examined by quantitative PCR with reverse 
a

NS

STZ

STZ-C

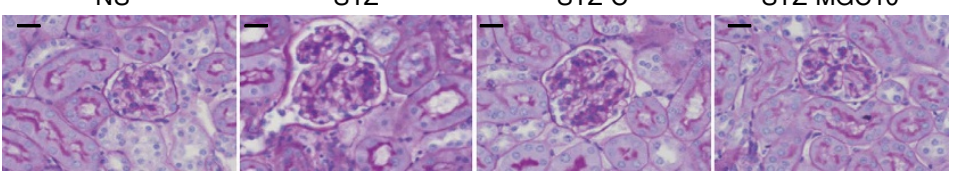

b
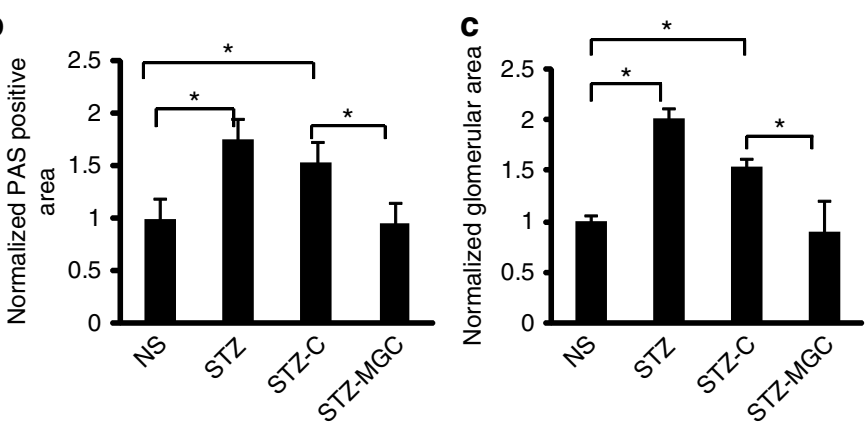

d
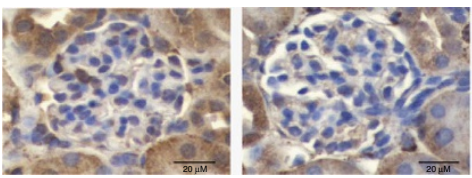

f

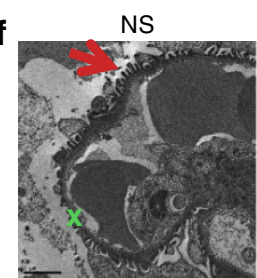

h
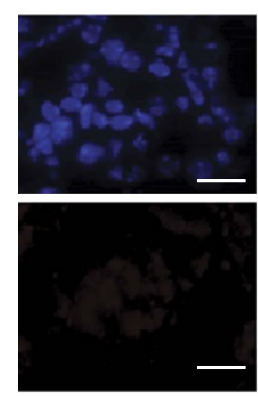

NS

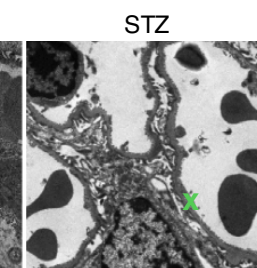

TZ
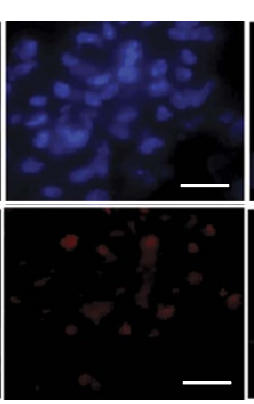

STZ

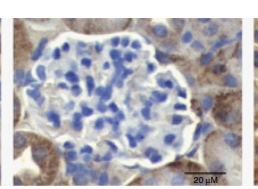

STZ-C
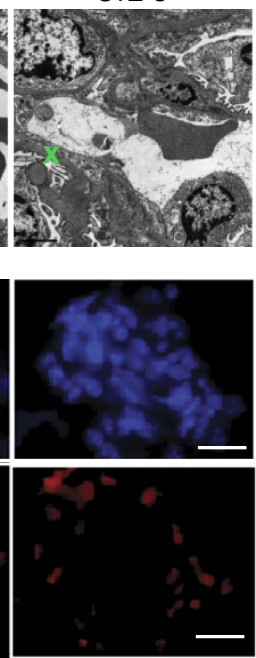

STZ-C

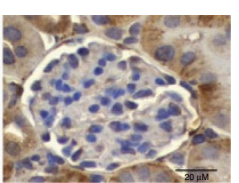

STZ-MGC10
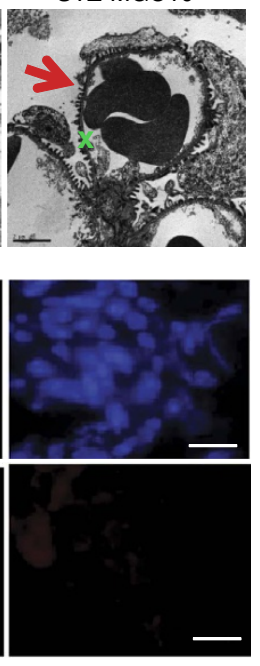

STZ-MGC10
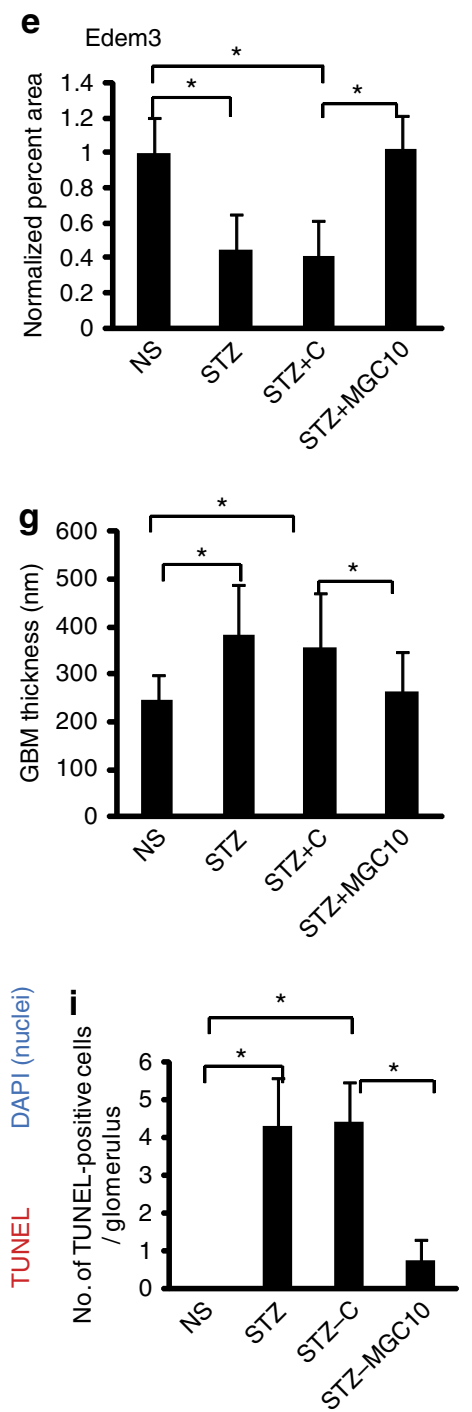

Figure 7 | In vivo effects of MGC10 on kidney pathology in diabetic mice. (a) PAS staining of kidney sections. Scale bar, $20 \mu \mathrm{m}$. (b,c) PAS positive area (b) and glomerular area (c) were significantly increased in diabetic mice (STZ and STZ-C) compared with non-diabetic mice (NS), and these were attenuated in diabetic mice injected with Gapmer targeting Inc-MGC, MGC10 (STZ-MGC10). (d) Edem3 staining in kidney sections. Scale bar, $20 \mu$ m. (e) Edem3 staining in kidney glomeruli was significantly decreased in diabetic mice (STZ and STZ + C) compared with non-diabetic mice (NS), and this was reversed in diabetic mice injected with MGC10 (STZ + MGC10). ${ }^{\star} P<0.05$. (f) EM structures of glomeruli. Distorted podocyte structure and increased GBM thickness were observed in glomeruli from diabetic mice as well as in diabetic mice injected with negative control GapmeR (STZ and STZ + C), but these features were attenuated in diabetic mice injected by GapmeR targeting Inc-MGC (STZ + MGC10). Red arrows denote intact podocytes. Green Xs denote a section of GBM. Scale bar, $2 \mu \mathrm{m}$. (g) GBM thickness was significantly increased in diabetic mice (STZ and STZ-C) compared with non-diabetic mice (NS), and this was attenuated in diabetic mice injected with MGC10 (STZ-MGC10). ${ }^{*} P<0.05$. (h) TUNEL assay. Top panels,

4,6-diamidino-2-phenylindole staining (blue) showing nuclei; second, TUNEL (red). $\times 400$ magnification. (i) Significant increase of TUNEL-positive cells was observed in diabetic mice (STZ and STZ-C) compared with non-diabetic mice (NS), and this was attenuated in diabetic mice injected with MGC10 (STZ-MGC10). ${ }^{\star} P<0.05$. Scale bar, $20 \mu \mathrm{m}$.

transcription (qRT-PCR) and small RNA sequencing in RNA isolated from microdissected glomeruli of kidney biopsies from 46 Southwestern American (Pima) Indians with documented type-2 diabetes ${ }^{57}$, as described ${ }^{17,58}$. The cluster miRNAs were expressed robustly in these diabetic patient samples with read frequency comparable to miR-192, which is highly enriched in the kidney and implicated in $\mathrm{DN}^{17,58}$ (Supplementary Table 1).
$\mathrm{DN}$ in humans is associated with glomerular hypertrophy, mesangial expansion and loss of podocytes leading to glomerulosclerosis ${ }^{2,6,59}$. We further found that increased expression of the precursors of some of the cluster miRNAs is significantly associated with morphometric parameters of increased glomerular damage (decreased podocyte density and increased podocyte and glomerular volume as well as mesangial index) in 

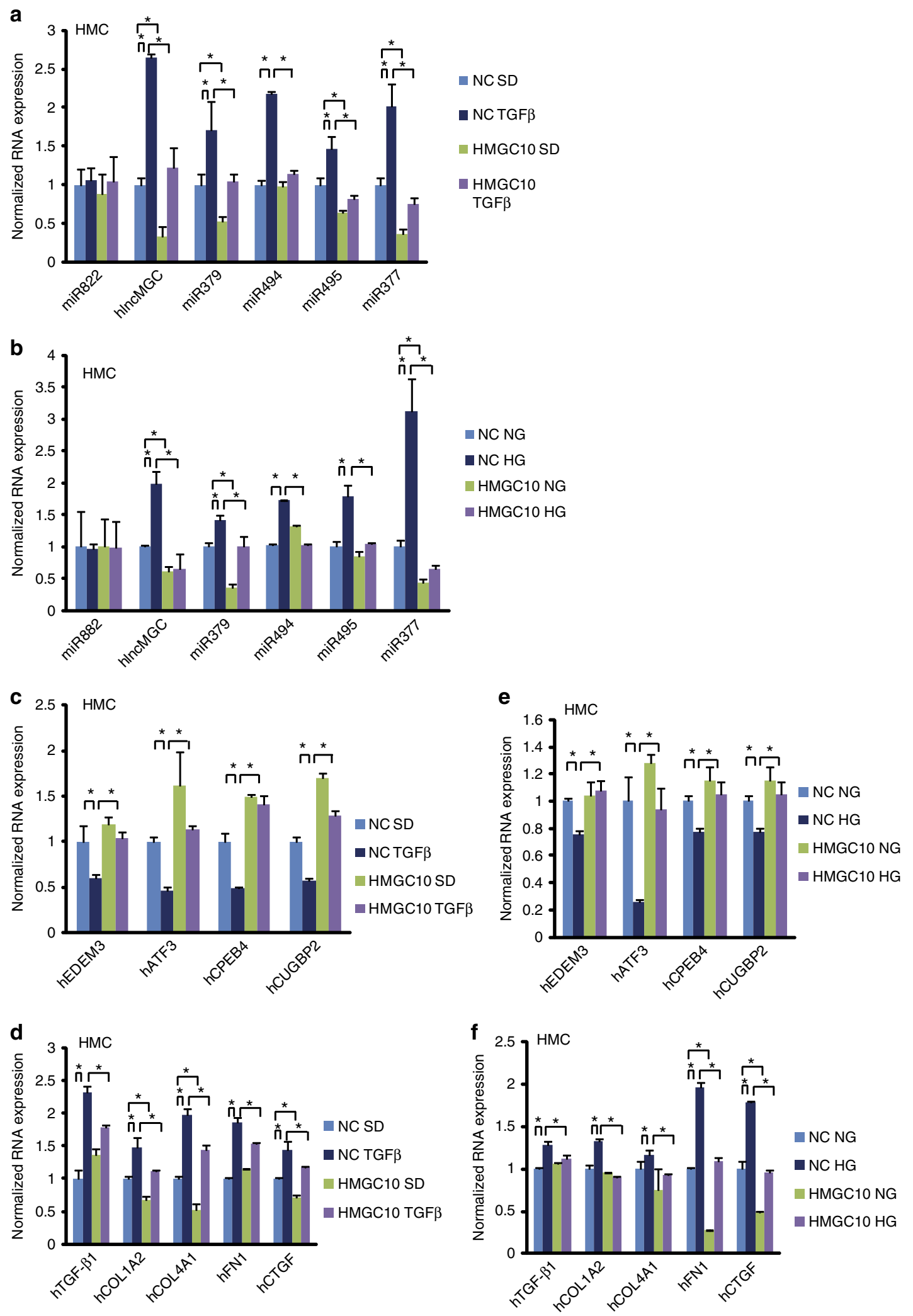

Figure 8 | Human homologue of Inc-MGC and its inhibition by HMGC10 in HMC. Significant increase of hlnc-MGC (human homologue of Inc-MGC), miR-379, miR-494, miR-495 and miR-377 in human MC (HMC) treated with TGF- $\beta 1$ (a) or HG (b) compared with respective controls (SD or normal glucose), but not miR-882 (outside of miR-379 cluster). These increases were significantly reduced in HMC transfected with HMGC10 compared with control oligo. (c) HMGC10 mediated restoration of miR-379 cluster targets, EDEM3, ATF3, CUGBP2 and CPEB4 which were inhibited by TGF- $\beta 1$ in HMC. (d) Significant increase of profibrotic genes, TGF- $\beta 1$, COL1A2, COL4A1, FN1 and CTGF in HMC treated with TGF- $\beta 1$ and their significant inhibition in HMC transfected with HMGC10. (e) HMGC10 mediated restoration of miR-379 cluster targets, EDEM3, ATF3, CUGBP2 and CPEB4 which were inhibited by HG in HMC. (f) Significant increase of profibrotic genes, TGF- $\beta 1$, COL1A2, COL4A1, FN1 and CTGF in HMC treated with HG which was significantly inhibited by HMGC10. Results are mean + s.e. in triplicate PCRs from three independent culture experiments. ${ }^{\star} P<0.05$. 
microdissected glomeruli of human nephrectomy tissues that showed various stages of glomerular pathology similar to early stages diabetic glomerulopathy ${ }^{60}$ (Supplementary Table 2). These associations suggest involvement of the megacluster miRNAs in human glomerular diseases including $\mathrm{DN}$, although additional studies are necessary.

\section{Discussion}

Our data suggest that diabetic conditions (HG), which also induce TGF- $\beta 1$, upregulates $\sim 40$ miRNAs within the miR-379 megacluster that target regulators of ER stress and protein synthesis resulting in hypertrophy and fibrosis related to DN (Fig. 9a). The host transcript (lnc-MGC) of this megacluster is also induced in parallel by Smad and CHOP, an ER stressresponsive transcription factor. miR-379 cluster expression depends on the expression of the host lnc-MGC from its promoter. siRNAs targeting Chop inhibited the induction of lnc-MGC and miR-379 cluster miRNAs as well as parameters (ECM gene expression and cellular hypertrophy) of $\mathrm{DN}$ in vitro. miRNAs in this cluster target several groups of genes, transcription factors, RNA binding proteins regulating fibrotic genes, as well as protein synthesis and ER stress, which result in hypertrophy via increased protein synthesis and fibrosis (ECM accumulation). Moreover, several cluster miRNAs had binding sites in the $3^{\prime}$ UTRs of multiple similar target genes. Although each cluster miRNA may have relatively modest effects by itself, the cumulative effects of upregulation of nearly 40 cluster miRNAs under diabetic conditions can have a considerable and synergistic impact to augment renal hypertrophy, fibrosis and dysfunction.

Induction of these miRNAs as well as features of early DN were ameliorated in the kidneys of diabetic Chop-KO mice compared to diabetic WT mice. Similarly, induction of these miRNAs and profibrotic genes by TGF- $\beta 1$ and HG were attenuated in MMC from Chop-KO mice. An ER stress inducer TM also induced lnc-MGC and cluster miRNAs in MMC likely through reduction of N-glycosylation of EDEM3. EDEM3 is reduced in pancreatic beta cells in type- 2 diabetes ${ }^{61}$. The Nephroseq database (http://www.nephroseq.org/) revealed correlations between GFR and EDEM3 expression in the normal human population, as well as in patients with DN, IgA nephropathy, and hypertensive nephropathy. Correlations of EDEM3 expression with obesity in human and mouse were also found in Nephroseq.

ER stress has been observed in patients with progressive $\mathrm{DN}^{62}$. Increased ER stress, renal Chop expression and albuminuria were reported in aged diabetic mice, and albuminuria was attenuated in diabetic Chop-KO mice compared to $\mathrm{WT}^{48}$. Reports showed increased levels of ER stress in animal models of $\mathrm{DN}^{46-51,63,64}$ and also amelioration of DN by ER stress inhibitors like 4-phenyl butyric acid $^{46}$ and the chemical chaperone TUDCA ${ }^{47}$. In the current study, we observed increase in Xbpl splicing which upregulates Chop (Supplementary Fig. 27e-g), but decreases in Atf 3 , a repressor of Chop as well as a direct target of miR-494 (refs 40-43) (an upregulated cluster miRNA) in MMC treated with HG or TGF- $\beta 1$ or in glomeruli from diabetic mice. The Nephroseq database also indicated lower levels of ATF3 in patients with DN compared to healthy controls. These data suggest two important ER stress-related signalling events, Xbp1 splicing and Atf 3 downregulation by miR-494, which co-operate to upregulate Chop in response to HG or TGF- $\beta 1$ in MMC (Fig. 9b). Therefore, ER stress is a major inducer of lnc-MGC in diabetes. Of course, CHOP can regulate other genes related to DN besides lnc-MGC, and also have cell-specific effects in the kidney ${ }^{64}$.

We further demonstrated that inhibition of the cluster miRNAs by a GapmeR (MGC10) which effectively targeted
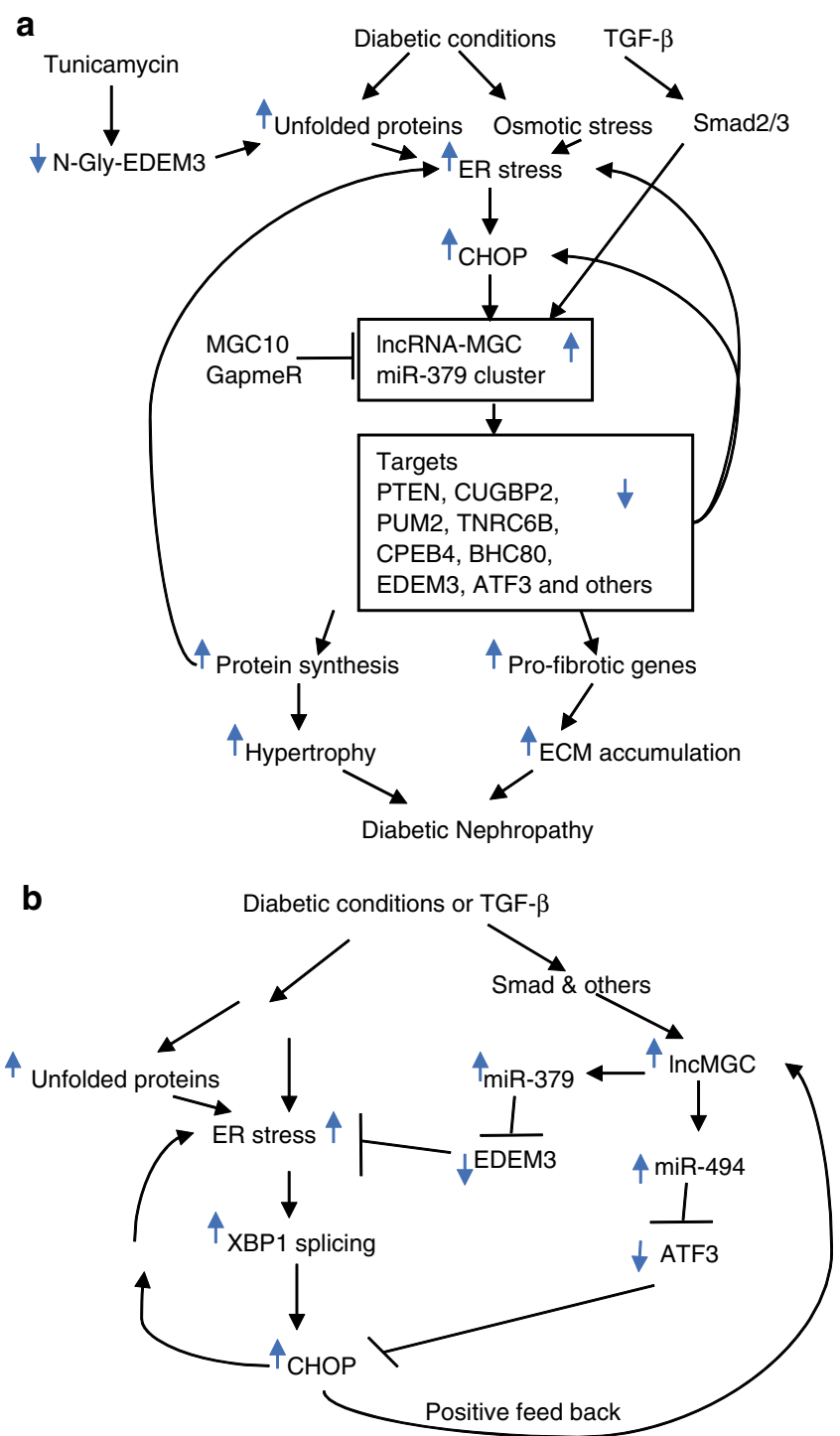

Figure 9 | ER-stress-regulated Inc-MGC hosting a miRNA cluster mediates features of DN. (a) Proposed model for the initiation of the early stages of DN through Inc-MGC, the miR-379 megacluster and related ER stress. Diabetic conditions and ensuing increases in TGF- $\beta 1$ can promote ER stress and induce Inc-MGC via Smad2/3 and CHOP. This results in increased expression of nearly 40 megacluster component miRNAs and subsequent downregulation of numerous targets whose functions are related to hypertrophy, ECM production and fibrosis associated with early features of DN. Targeting Inc-MGC in vivo with specific LNA GapmeRs can attenuate these pathological events, and thereby prevent further disease progression. Please see main text for additional details. (b) A schematic model of CHOP upregulation through two pathways: first, XBP-1 splicing activated by ER stress; and second, reduction in levels of ATF3 (a repressor of $(\mathrm{HOP}$ ) due to targeting by the cluster miR-494 in MC treated with TM, $\mathrm{HG}$ or TGF- $\beta 1$. CHOP in turn can induce Inc-MGC and ER stress in positive feedback loops.

lnc-MGC, ameliorated key features of early DN (ECM accumulation and glomerular hypertrophy) in a mouse model. Since this lncRNA-miRNA cluster is induced early in the disease process and controls several critical mechanisms associated with the initial stages of $\mathrm{DN}$, reducing its expression can prevent further progression of $\mathrm{DN}$. In early $\mathrm{DN}, \mathrm{MC}$ are a major source of increased TGF- $\beta 1$ production ${ }^{65}$. HG induces TGF- $\beta 1$ in MC through promoter E-boxes ${ }^{16,66}$. However, HG induces only 
TGF- $\beta$ RII (receptor) but not TGF- $\beta 1$ in podocytes ${ }^{67}$. TGF- $\beta 1$ secreted by $\mathrm{MC}$ may have autocrine effects which activate $\mathrm{MC}$, and paracrine effects which activate other cells including podocytes. This suggests that events occurring in MC (induction of TGF- $\beta 1$ ) are earlier than those in podocytes (podocyte loss induced by diabetes and TGF- $\beta 1$ ). Significant podocyte loss was detected at 20 weeks after the onset of diabetes in mice, while podocyte injury, effacement and death were detected at 4 weeks which can be related to subsequent proteinuria ${ }^{56}$. However, induction of TGF- $\beta 1$ and ECM accumulation in MC were detected by 2 weeks after the onset of diabetes ${ }^{12,17}$. Therefore, $\mathrm{MC}$ injury and ECM accumulation are most likely initial events in glomeruli, with increased TGF- $\beta 1$ secreted by MC triggering subsequent podocyte injury/death soon after ${ }^{65}$. Furthermore, other factors related to DN (HG, advanced glycation end-products, lipids, Angiotensin II) can also promote ER stress and subsequently induce lnc-MGC via TGF- $\beta 1$ dependent and independent pathways. Therefore, strategies, such as those described here, targeting early events in the glomeruli (MC and podocyte injury) can prevent $\mathrm{DN}$ progression. This is supported by our data showing GapmeR MGC10 attenuates not only the increases in glomerular mesangial hypertrophy/expansion, fibrosis and GBM thickening in diabetic mice at 4 weeks, but also the increases in podocyte effacement and cell death.

Notably, key cluster miRNAs as well as hlnc-MGC were also upregulated in HMC treated with HG or TGF- $\beta 1$ and the corresponding targets were downregulated. A GapmeR targeting hlnc-MGC ameliorated these events, as well as the induction of fibrotic genes. Most of the cluster miRNAs were expressed in microdissected glomeruli obtained from diabetic Pima Indians, and the expression of some of their precursors were increased with glomerular damage, suggesting relevance for human glomerular diseases.

Together, these results demonstrate new functions for a lncRNA and its component miRNAs in DN and possibly other glomerular diseases. Several reports have demonstrated that inhibition of specific miRNAs by modified oligonucleotides ameliorated DN in animal models $s^{6,10-12,16-21}$. However, to our knowledge, this is the first report that a single oligonucleotide (GapmeR) targeting a lncRNA which hosts a cluster of miRNAs can control the expression of the host IncRNA, component miRNAs, their targets, profibrotic genes and symptoms of DN in mice, and in human MC.

\footnotetext{
Methods

Mouse models of diabetes. All animal studies were conducted according to a protocol approved by the Institutional Animal Care and Use Committee at the Beckman Research Institute of City of Hope. Male type-2 diabetic $d b / d b$ mice (T2D leptin receptor deficient; Strain BKS.Cg-m $+/+$ lepr $\mathrm{db} / \mathrm{J}$ ) and genetic control non-diabetic $d b /+$ mice (10-12 weeks old), were obtained from The Jackson Laboratory (Bar Harbor, ME) ${ }^{11,17}$. Male C57BL/6 mice (10 week old, The Jackson Laboratory) were injected with $50 \mathrm{mg} \mathrm{kg}^{-1}$ of STZ intraperitoneally on 5 consecutive days. Mice injected with diluent served as controls. Diabetes was confirmed by tail vein blood glucose levels (fasting glucose $>300 \mathrm{mg} \mathrm{dl}^{-1}$ ). Each group was composed of five to six mice. Mice were sacrificed at 4-5 or 22 (ref. 17) weeks post-induction of diabetes. Glomeruli were isolated from freshly harvested kidneys by a sieving technique ${ }^{11,17}$ in which renal capsules were removed, and the cortical tissue of each kidney separated by dissection. The cortical tissue was then carefully strained through a stainless sieve with a pore size of $150 \mu \mathrm{m}$ by applying gentle pressure. Enriched glomerular tissue below the sieve was collected and transferred to another sieve with a pore size of $75 \mu \mathrm{m}$. After several washes with cold PBS, the glomerular tissue remaining on top of the sieve was collected. Pooled glomeruli were centrifuged, and the pellet was collected for RNA, protein extraction or for preparing MMCs ${ }^{11,17}$. Male Chop-KO mice were also obtained from the Jackson Laboratory (B6.129S(Cg)-Ddit $\left.{ }^{\text {tm } 2.1 D r o n} / \mathrm{J}\right)$. Based on our previous experience, sample size was determined to have enough power to detect an estimated difference between two groups. With minimum sample size of 5 in each group, the study can provide at least $80 \%$ power to detect an effect size of 2 between diabetic and non-diabetic groups or treated and untreated groups at the
}

0.05 significant level using two-sided $t$-test. Since we expected larger variation between groups especially for the mice with oligo-injection, we used more than 5 mice in each group (with 6 mice in each group, we have $80 \%$ power to detect an effect size of 1.8 at the 0.05 confidence level). Our actual results with current sample size did show statistical significance for majority of the miRNAs in the cluster. Histopathological and biochemical analysis of tissues or cells derived from animal models were performed by investigators masked to the genotypes or treatments of the animals.

Primary mesangial cells. MMC were obtained from glomeruli and cultured in vitro ${ }^{11,28}$. Glomeruli isolated from freshly harvested kidneys were incubated with $0.1 \mathrm{mg} \mathrm{ml}^{-1}$ collagenase at $37^{\circ} \mathrm{C}$ in shaking water bath for $15 \mathrm{~min}$. After incubation with shaking, cells were cultured in Roswell Park Memorial Institute medium supplemented with $20 \%$ foetal bovine serum and $20 \mu \mathrm{g} \mathrm{ml}^{-1}$ Insulin. After 2-3 passages, MMC were maintained in Roswell Park Memorial Institute medium 1640 medium supplemented with $10 \%$ foetal bovine serum. Passages $5-7$ were used for experiments.

Human MCs (CC-2559, 47 Y Male) were purchased from Lonza Walkersville, Inc. and cultured as per the instructions of the provider.

Small RNA sequencing. Total RNA was used for the construction of small RNA libraries, cluster generation and then deep sequencing (at the Beckman Research Institute Integrative Genomics Core) using the Alternative v1.5 Protocol (Illumina Inc., San Diego, CA) with minor optimization as described previously ${ }^{22}$. Briefly, $0.5 \mu \mathrm{g}$ of total RNA was ligated to sRNA $3^{\prime}$ adaptor (5'-AUCUCGUAUGCCGU CUUCUGCUUG- $3^{\prime}$ ) using T4 RNA Ligase 2, truncated (New England BioLabs, Ipswich, MA) at $22^{\circ} \mathrm{C}$ for $1 \mathrm{~h}$, and subsequently ligated to the SRA $5^{\prime}$ adaptor ( $5^{\prime}$-GUUCAGAGUUCUACAGUCCGACGAUC- $3^{\prime}$ ) with T4 RNA ligase (New England BioLabs, Ipswich, MA) at $20^{\circ} \mathrm{C}$ for $1 \mathrm{~h}$. The adaptor-linked RNA was converted to single-stranded cDNA using Superscript II reverse transcriptase (Invitrogen) and RT-Primer (5'-CAAGCAGAAGACGGCATACGA-3') and then amplified with Phusion DNA Polymerase (Finnzymes Thermo Scientific, Pittsburgh, PA) for 12 cycles using primers (5'-CAAGCAGAAGACGGCATA CGA-3'; 5'-AATGATACGGCGACCACCGACAGGTTCAGAGTTCTACAGTC CGA-3'). PAGE purification was carried out to select small RNAs of 17-52 nucleotides in length. The purified library was quantified using qPCR, used for cluster generation on cBot, and sequenced using Genome Analyzer IIx (Illumina).

Analysis of small RNA sequencing data. For analyses of small RNA sequencing data $^{22}$, raw data in FASTQ format generated from the Illumina pipeline was aligned against UCSC mouse assembly $\mathrm{mm} 9$ using Novoalign software. The 3 -adaptor sequence of the raw reads was first trimmed by Novoalign, and reads with 16 or more bases were aligned to the genome. For reads aligned to multiple locations on the genome, one aligned region was randomly selected for counting the number of reads as described ${ }^{22}$. Genomic locus of each mouse mature miRNA was generated by aligning mouse mature miRNA sequences (miRBase, www.mirbase.org) without allowing mismatches. For each sample, the reads corresponding to the mature miRNA genomic loci (including 10-base flanking regions) were counted to obtain expression levels of total miRNAs. The resulting miRNA expression data set was further normalized by scaling the total mature miRNA counts in each sample to 6 million. miRNAs with scaled reads of no $<5$ in either sample were considered as detectable ones. Among them, differentially expressed miRNAs were identified with at least twofold change in glomeruli from STZ typel diabetic mice versus non-diabetic mice. Pre-ranked GSEA (ref. 24) were conducted on miR-379 cluster miRNAs using all the detectable miRNAs ranked by $\log 2$ fold changes between the two samples. For each miR-379 cluster miRNA, the potential human or mouse target genes containing at least 3 conserved binding sites were identified using the online bioinformatics tool TargetScan (http:// www.targetscan.org/). The human/mouse target genes were then pooled together to generate human/mouse gene set. IPA was applied to each gene set for gene ontology, pathway and network analyses.

miRNAs and siRNAs. Oligonucleotide mimics of miRNAs, siRNAs and corresponding control oligos were obtained from integrated DNA technologies or Thermo Fisher Scientific Inc. (Waltham, MA), as described ${ }^{11,28}$. Wild-type (WT) MMCs (from WT C57BL/6 mice), MMC transfected with Chop siRNA, and MMCs from Chop-KO mice were treated with HG, mannitol and TGF- $\beta 1$ as described $^{1,28}$. Briefly, cells $\left(\sim 10^{6} /\right.$ transfection) were transfected with siRNA or miRNA oligonucleotides using an Amaxa Nucleofector (Lonza, Basel, Switzerland) according to the manufacturer's protocols ${ }^{11,28}$. siRNAs (double-stranded oligos of three pairs of sense (S) and antisense (AS) synthesized oligos,

S1 rCrArUrCrUrGrCrUrUrCrCrCrArCrUrGrCrCrArArArUrCAG and AS1 rCrUrGrArUrUrUrGrGrCrArGrUrGrGrGrArArGrCrArGrArUrGrUrG; S2 rUrCrArGrCrArCrCrGrUrGrCrArArCrCrArUrUrCrArArGGA and AS2 rUrCrCrUrUrGrArArUrGrGrUrUrGrCrArCrGrGrUrGrCrUrGrArArA; S3 rCrUrUrCrArUrCrUrGrGrUrArArUrGrUrArCrUrArCrCrUGA and AS3 rUrCrArGrGrUrArGrUrArCrArUrUrArCrCrArGrArUrGrArArGrGrC; (r, ribose) against mouse upstream region of miR-379 were obtained from integrated DNA technologies. Non-targeting siRNA controls were obtained from Thermo Fisher 
Scientific Inc. MMC were trypsinized and resuspended in basic nucleofection solution at $1 \times 10^{7} \mathrm{ml}^{-1}$. Subsequently, $100 \mu$ of cell suspension $\left(1 \times 10^{6}\right.$ cells $)$ was mixed with miRNA mimic, hairpin inhibitor oligonucleotides, or ON-TARGET plus siRNA or negative controls (Thermo Fischer Scientific Inc., Waltham, MA). Transfected cells were harvested for RNA and protein extraction. RNA was extracted from the cells and the expression of IncRNA-MGC, and miRNAs within the cluster were examined using primers designed for each of the mature miRNAs (miRBase). MMCs were transfected with oligonucleotide mimics, siRNAs of candidate miRNAs or negative control (NC) oligos to determine if manipulating their levels can influence TGF- $\beta 1$ and HG responses. At $48-72 \mathrm{~h}$ post-transfection, the expression of miRNA target genes, fibrosis and hypertrophy related genes, and proteins induced by TGF- $\beta 1 /$ HG were determined by RT-qPCR and western blotting using our published methods ${ }^{11,45}$

Real-time qPCR. Real-time qRT-PCR analysis was performed as previously described $^{11,45}$. Briefly, RNA was extracted using miRNeasy columns (Qiagen, Valencia, CA). miRNA expression analysis was performed using the qScript miRNA cDNA synthesis kit (Quanta Biosciences, Gaithersburg, MD) and PerfeCTa SYBR Green Supermix (Quanta Biosciences). miRNAs were amplified using specific mature miRNA sequences as forward primers and the universal primer provided in the kit as the reverse primer. U6 was used as internal control. A GeneAmp RNA PCR kit (Applied Biosystems, Carlsbad, CA) and POWER SYBR Green mix (Applied Biosystems) were used for mRNA quantification. PCR primer sequences are in Supplementary Table 3.

Western blotting. Immunoblotting was performed as described previously 11,28 Cells were lysed in Laemmli's sample buffer. Lysates were fractionated on $10 \%$ SDS-polyacrylamide gels (Bio-Rad) and transferred to nitrocellulose membrane. Membranes were immunoblotted with appropriate antibodies. Blots were scanned using GS-800 densitometer and quantified with Quantity One software (Bio-Rad). Wider (uncropped) scans of blots are shown in Supplementary Figs 38 and 39. Antibodies used were $\beta$-actin (Sigma, A-5441), CHOP (Santa Cruz Biotechnology, sc-793), ZEB1(Santa Cruz Biotechnology, sc-25388), USF1(Santa Cruz Biotechnology, sc-229), EDEM3 (Abcam, ab67108), BHC60 (Santa Cruz Biotechnology, sc-109846), CPEB4 (abcam, ab83009), Tfe3 (BD Bioscience, cat\# 554263) and CUGBP2 (GenWay Biotech, Inc., cat\# 18-003-43569). Antibodies were used at 1:1,000 dilution.

Rapid amplification of $5^{\prime}$ complementary DNA ends (5' RACE). We used $5^{\prime}$ RACE to identify the putative upstream promoter region of the miR-379 megacluster and host lncRNA-MGC. $5^{\prime}$ RACE was performed using GeneRacer kit (Invitrogen) using RACE primers, miR379upR1, 5'-GACCCCTAGAAACTTGG GCCTTGTCCCTG- $3^{\prime}$ and miR379upR2, 5' -TCAGGAACCATGGAACGGTGTT GACCCCTAG- $3^{\prime}$. Amplified PCR fragments were cloned and sequenced and the transcription start site determined using RNAs from glomeruli from diabetic mice (STZ). To amplify the upstream region, primer miR379upINR including potential initiator (INR), $5^{\prime}$-ATTTTTCTGAGTTAGTGTGGCCTTCATCTG-3' was used.

Reporter assays. Mouse Edem $33^{\prime}$ UTR reporter plasmid was purchased from OriGene (Rockville, MD). Deletion mutants of Edem $33^{\prime} \mathrm{UTR}$ were generated by digesting full-length $3^{\prime} \mathrm{UTR}$ with EcoRI and re-ligation. Full-length and deletion mutants of reporter plasmids were transfected into MMC and treated with or without TGF- $\beta 1\left(10 \mathrm{ng} \mathrm{ml}^{-1}, 6-24 \mathrm{~h}\right)^{11,28}$. The fragment of upstream of lnc-MGC (miR-379 cluster) digested with SacI and HindIII was cloned into pGL4 luciferase vector (Promega). CHOP binding site mutant was created by PCR using CHOP site mutant primer $5^{\prime}$-GAGCTCTgcGCTCTgcGCACCTGCGCTT- $3^{\prime}$ and miR379upR1, $5^{\prime}$-GACCCCTAGAAACTTGGGCCTTGTCCCTG-3' ${ }^{\prime}$ and SacI/HindIII digested fragment was cloned into pGL4.

Chromatin immunoprecipitation assays. ChIP assays were performed as previously described ${ }^{11,28}$. Briefly, MMCs were treated with TGF- $\beta 1\left(10 \mathrm{ng} \mathrm{ml}^{-1}\right)$ or HG $(25 \mathrm{mM})$ and then cross-linked with formaldehyde. The cross-linked chromatin was sheared and immunoprecipitated with antibodies $\left(\sim 10 \mu \mathrm{g} \mathrm{ml}^{-1}\right)$ against Smad2/3, CHOP, USF1, ZEB1 or TFE3. ChIP-enriched DNA was purified and used as a template for real-time qPCR; the CHOP binding element in the promoter region of lnc-MGC was amplified with following primers: forward 5'- GAGCTCTTGCTCTTTGCACCTGCG-3' and reverse $5^{\prime}$ - AAGCAGGTGGA ACCAGAAGTAAGCC-3'. ChIP-qPCR results (normalized enrichment) were calculated by the $2-\Delta \Delta C_{t}$ method (where $C_{t}$ is threshold cycle) and normalized to input DNA (purified from $5 \%$ of the same cross-linked chromatin).

GapmeRs. GapmeRs are antisense oligonucleotides having a central stretch of DNA that is complementary to the target which is flanked by LNAs, and they induce RNAse $\mathrm{H}$ mediated target degradation. Twenty GapmeRs were designed in silico to inhibit the expression of lnc-MGC. These 20 GapmeRs fell into four clusters and four representative GapmeRs (one from each cluster) were synthesized and obtained from Exiqon (Vedbaek, Denmark) (MGC1, TCAaaaacataacGCC;
MGC5, CACggtgctgaaaGAG; MGC8, TGAaggccacactAAC; MGC10, ATTtggcag tgggAAG, uppercase: LNA; lowercase: DNA, full phosphorothioate). We used a well-established and characterized control oligo provided by Exiqon which has the same modification as our IncRNA targeting GapmeRs and has no homology to any known mRNA, miRNA or lncRNA in mouse, rat and human (negative control A AACacgtctataCGC). One of the most consistently effective oligos identified by in vitro screening was MGC10, while other GapmeRs had lesser effects on the expression of lnc-MGC. 2 or $5 \mathrm{mg} \mathrm{kg}^{-1}$ MGC10 was injected subcutaneously into control and STZ-injected diabetic C57BL6 mice. Human version of MGC10 (HMGC10) was designed based on the human sequence. HMGC10, (GATttggcattggAAG; uppercase: LNA; lowercase: DNA, full phosphorothioate).

Serum toxicity analyses. Serum samples from the mice were sent to the IDEXX laboratory (Irvine, CA) for Preclinical Research Services (clinical biochemistry panels to evaluate liver and kidney toxicity).

Serum cytokine profiles. Cytokine profiles in the mice sera were examined using a pre-mixed mouse 10-plex panel from Invitrogen that includes: GM-CSF, INF-g, IL-1b, IL-2, IL-4, IL-5, IL-6, IL-10, IL-12(P40/P70) and TNF- $\alpha$ at the Analytical Pharmacology Core Facility at City of Hope.

In situ hybridization. To detect LNA-GapmeR MGC10 accumulation in vivo, in situ hybridization was performed as described previously ${ }^{11}$. Briefly, frozen kidney cortex sections from LNA-MGC10 injected and saline control mice $(n=3$ per group) were fixed in paraformaldehyde ( $3 \%$ for $10 \mathrm{~min}$ at room temperature) followed by rinsing in PBS. Slides were pre-hybridized (at $48^{\circ} \mathrm{C}$ for $30 \mathrm{~min}$ ) in hybridization solution ( $50 \%$ formamide, $5 \times$ saline sodium citrate buffer (SSC), $500 \mu \mathrm{g} \mathrm{ml}^{-1}$ yeast transfer RNA and $1 \times$ Denhardt's solution), and then hybridized (at $48^{\circ} \mathrm{C}$ for $5 \mathrm{~min}$ ) with a TEX615 Red (Exiqon) fluorescent-labelled LNA-modified oligonucleotide probe complementary to LNA-MGC10 (16mer, 5'-TEX615-cTtcCcaCtgCcaAat-3' (uppercase: LNA; lowercase: DNA; Exiqon) followed by post-hybridization wash three times at $55^{\circ} \mathrm{C}$ in $0.1 \times$ SSC. Slides were then stained with 4,6-diamidino-2-phenylindole, mounted and analysed using a fluorescence microscope.

Histology and immunohistochemistry. Formalin-fixed, paraffin-embedded sections of mouse kidneys were mounted onto positively charged slides, deparaffinized, washed with water, blocked with Dako protein block (Dako, Carpinteria, CA), and incubated with EDEM3 antibody for 30 min. Slides were washed with Dako wash, treated with hydrogen peroxide for $5 \mathrm{~min}$, washed with PBS, incubated with anti-rabbit secondary antibody conjugated with a peroxidase polymer (Dako, Carpinteria, CA), and washed and incubated with 3,3'-diaminobenzidine for $8 \mathrm{~min}$. Slides were counterstained with haematoxylin and mounted. PAS staining was performed to analyse ECM deposition. Images were taken using Olympus BX51 microscope with In Studio (Pixera Corp., Santa Clara, CA) software to collect images. ImagePro software (Media Cybernetics Inc., Rockville, MD) was used to quantify staining.

TUNEL assay. Combined TUNEL in situ staining and WT1 (Wilms Tumor) immunohistochemical staining was performed on frozen kidney sections from control and diabetic mice as well as mice groups injected with control oligo and MGC10. The in situ Cell Death Detection (TMR red) kit was purchased from Roche and WT1 antibody was purchased from Santa Cruz. Briefly, $5 \mu \mathrm{m}$ sections were fixed and processed for TUNEL staining according to manufacturer's instructions. The slides were then washed with PBS and processed for immunohistochemical staining of WT1 and mounted using Prolong Gold antifade reagent with 4,6-diamidino-2-phenylindole (Applied Biosystems) for visualization.

Electron microscopy. EM structures of mouse kidney glomeruli were evaluated in the EM core of City of Hope. Paraffin-embedded tissue was deparaffinized with xylene, rehydrated in a graded ethanol series to distilled water and fixed with $2 \%$ glutaraldehyte in $0.1 \mathrm{M}$ Cacodylate buffer $\left(\mathrm{Na}\left(\mathrm{CH}_{3}\right)_{2} \mathrm{AsO}_{2} 3 \mathrm{H}_{2} \mathrm{O}\right)$, pH7.2, at $4{ }^{\circ} \mathrm{C}$, overnight. The samples were washed three times with $0.1 \mathrm{M}$ Cacodylate buffer, $\mathrm{pH} 7.2$, post-fixed with $1 \% \mathrm{OsO}_{4}$ in $0.1 \mathrm{M}$ Cacodylate buffer for $30 \mathrm{~min}$ and washed three times with $0.1 \mathrm{M}$ Cacodylate buffer. The samples were then dehydrated through $60 \%, 70 \%, 80 \%, 95 \%$ ethanol, $100 \%$ absolute ethanol (twice), propylene oxide (twice) and were left in propylene oxide/Eponate (1:1) overnight at room temperature. The vials were sealed. The next day, the vials were left open for $2-3 \mathrm{~h}$ to evaporate the propylene oxide. The samples were infiltrated with $100 \%$ Eponate and polymerized at $\sim 64^{\circ} \mathrm{C}$ for $48 \mathrm{~h}$. For EM, ultra-thin sections ( $\sim 70 \mathrm{~nm}$ thick) were cut using a Leica Ultra cut UCT ultramicrotome with a diamond knife, picked up on 200 mesh copper EM grids. Grids were stained with $2 \%$ uranyl acetate for 10 min followed with Reynold's lead citrate staining for 1 min. EM was done on an FEI Tecnai 12 transmission electron microscope equipped with a Gatan Ultrascan $2 \mathrm{~K}$ CCD camera. ImagePro software (Media Cybernetics Inc., Rockville, MD) was used to quantify GBM thickness, the distance between the endothelial and podocyte plasma membranes. More than 20 measurements were made in each group to determine an average GBM thickness. 
Measurement of cellular hypertrophy. Hypertrophy was assessed by measurement of cellular protein/cell counts ${ }^{11}$. MMC were trypsinized and counted using a Coulter Counter with 100- $\mu \mathrm{m}$ aperture (Beckman Coulter, Brea, CA). Cells were lysed, and total protein content was measured using protein assay kit from Bio-Rad.

Human sample studies. Cluster miRNA expression was determined by small RNA sequencing in microdissected glomeruli from adult Pima Indians with type 2 diabetes who participated in a 6-year clinical trial to evaluate the renoprotective efficacy of losartan (ClinicalTrials.gov number NCT00340678) ${ }^{57}$. Of the 169 clinical trial participants, 121 underwent kidney biopsy and 46 of the participants who underwent kidney biopsies were included in the present study. This study was approved by the Institutional Review Board of the National Institute of Diabetes and Digestive and Kidney Diseases. The expression of cluster miRNA precursors was associated with morphometric parameters in 39 human nephrectomy samples. Age ranged from 31 to 85 years. Morphometric analysis was performed in formalin-fixed paraffin-embedded tissue sections combining PAS staining and WT1 as podocyte marker with quantitative image analysis as described ${ }^{58,60}$ This study was approved by the Institutional Review Board of the University of Michigan. Each subject gave written informed consent.

Quantification of miRNAs from human renal biopsies. Glomeruli were microdissected from kidney biopsies, and nephrectomy tissue samples submerged in RNAlater manually using a dissection microscope. Total RNA was isolated using spin-columns; miRNA expression was quantified using qRT-PCR performed using TaqMan Array Human MicroRNA Card (Applied Biosystems) and small RNA-sequencing ${ }^{58}$. Samples were normalized to geometric mean of reference RNAs (refs 17,58). Expression of miRNA precursors was determined in microdissected glomeruli of nephrectomy samples using Affymetrix Human Gene 2.1 ST 24-Array ${ }^{58,68}$.

Statistical analysis. Normal distribution of each sample group was confirmed by $\chi^{2}$ test before comparison between groups. Statistical analyses were performed by Student's $t$-tests (two-sided) to compare two groups (planned comparison) and Benjamini-Hochberg adjusted for multiple comparisons $s^{69}$. If variances between two groups were significantly different (F-test), $t$-test assuming unequal variances was used in the comparison. $P<0.05$ was considered as statistically significant. Power analyses for the animal experiments have been described earlier.

Data availability. The authors declare that all data supporting the findings of this study are available within the article and its Supplementary Information Files or are available from the corresponding authors on request. The sequencing data sets (including both raw sequences and processed data) have been deposited in the NCBI Gene Expression Omnibus (GEO) database (GEO accession number GSE69718, https://www.ncbi.nlm.nih.gov/geo/query/acc.cgi?acc=GSE69718).

\section{References}

1. Kanwar, Y. S., Sun, L., Xie, P., Liu, F. Y. \& Chen, S. A glimpse of various pathogenetic mechanisms of diabetic nephropathy. Annu. Rev. Pathol. 6, 395-423 (2011).

2. Kato, M. \& Natarajan, R. MicroRNAs in diabetic nephropathy: functions, biomarkers, and therapeutic targets. Ann. N. Y. Acad. Sci. 1353, 72-88 (2015).

3. Sharma, K. \& Ziyadeh, F. N. Hyperglycemia and diabetic kidney disease. The case for transforming growth factor-beta as a key mediator. Diabetes $\mathbf{4 4}$, 1139-1146 (1995).

4. Ziyadeh, F. N. et al. Long-term prevention of renal insufficiency, excess matrix gene expression, and glomerular mesangial matrix expansion by treatment with monoclonal antitransforming growth factor-beta antibody in $\mathrm{db} / \mathrm{db}$ diabetic mice. Proc. Natl Acad. Sci. USA 97, 8015-8020 (2000).

5. Yamamoto, T., Nakamura, T., Noble, N. A., Ruoslahti, E. \& Border, W. A. Expression of transforming growth factor beta is elevated in human and experimental diabetic nephropathy. Proc. Natl Acad. Sci. USA 90, 1814-1818 (1993).

6. Kato, M. \& Natarajan, R. Diabetic nephropathy--emerging epigenetic mechanisms. Nat. Rev. Nephrol. 10, 517-530 (2014).

7. Reidy, K., Kang, H. M., Hostetter, T. \& Susztak, K. Molecular mechanisms of diabetic kidney disease. J. Clin. Invest. 124, 2333-2340 (2014).

8. Massague, J. TGFbeta signaling: receptors, transducers, and Mad proteins. Cell 85, 947-950 (1996).

9. Bartel, D. P. MicroRNAs: target recognition and regulatory functions. Cell 136, 215-233 (2009)

10. Trionfini, P., Benigni, A. \& Remuzzi, G. MicroRNAs in kidney physiology and disease. Nat. Rev. Nephrol. 11, 23-33 (2015).

11. Kato, M. et al. TGF-beta activates Akt kinase through a microRNA-dependent amplifying circuit targeting PTEN. Nat. Cell Biol. 11, 881-889 (2009).

12. Putta, S. et al. Inhibiting microRNA-192 ameliorates renal fibrosis in diabetic nephropathy. J. Am. Soc. Nephrol. 23, 458-469 (2012).
13. Krutzfeldt, J. et al. Silencing of microRNAs in vivo with 'antagomirs'. Nature 438, 685-689 (2005).

14. van Rooij, E. \& Kauppinen, S. Development of microRNA therapeutics is coming of age. EMBO Mol. Med. 6, 851-864 (2014).

15. Lindow, M. \& Kauppinen, S. Discovering the first microRNA-targeted drug. J. Cell Biol. 199, 407-412 (2012).

16. Kato, M. et al. A microRNA circuit mediates transforming growth factor-betal autoregulation in renal glomerular mesangial cells. Kidney Int. 80, 358-368 (2011).

17. Deshpande, S. D. et al. Transforming growth factor-beta-induced cross talk between p53 and a microRNA in the pathogenesis of diabetic nephropathy. Diabetes 62, 3151-3162 (2013).

18. Long, J., Wang, Y., Wang, W., Chang, B. H. \& Danesh, F. R. MicroRNA-29c is a signature microRNA under high glucose conditions that targets Sprouty homolog 1, and its in vivo knockdown prevents progression of diabetic nephropathy. J. Biol. Chem. 286, 11837-11848 (2011).

19. He, F. et al. MiR-135a promotes renal fibrosis in diabetic nephropathy by regulating TRPC1. Diabetologia 57, 1726-1736 (2014).

20. Zhong, X. et al. miR-21 is a key therapeutic target for renal injury in a mouse model of type 2 diabetes. Diabetologia 56, 663-674 (2013).

21. Chau, B. N. et al. MicroRNA-21 promotes fibrosis of the kidney by silencing metabolic pathways. Sci. Transl. Med. 4, 121 ral18 (2012).

22. Jin, W. et al. Small RNA sequencing reveals microRNAs that modulate angiotensin II effects in vascular smooth muscle cells. J. Biol. Chem. 287, 15672-15683 (2012).

23. Kameswaran, V. et al. Epigenetic regulation of the DLK1-MEG3 microRNA cluster in human type 2 diabetic islets. Cell Metab. 19, 135-145 (2014).

24. Subramanian, A. et al. Gene set enrichment analysis: a knowledge-based approach for interpreting genome-wide expression profiles. Proc. Natl Acad. Sci. USA 102, 15545-15550 (2005).

25. Wang, K. C. et al. A long noncoding RNA maintains active chromatin to coordinate homeotic gene expression. Nature 472, 120-124 (2011).

26. Wang, Q. et al. MicroRNA-377 is up-regulated and can lead to increased fibronectin production in diabetic nephropathy. FASEB J 22, 4126-4135 (2008).

27. Kriegel, A. J. et al. MicroRNA-target pairs in human renal epithelial cells treated with transforming growth factor beta 1: a novel role of miR-382. Nucleic Acids Res. 38, 8338-8347 (2010).

28. Kato, M. et al. TGF-beta induces acetylation of chromatin and of Ets-1 to alleviate repression of miR-192 in diabetic nephropathy. Sci. Signal. 6, ra43 (2013).

29. Alvarez, M. L., Khosroheidari, M., Eddy, E. \& Kiefer, J. Role of microRNA 1207-5P and its host gene, the long non-coding RNA Pvt1, as mediators of extracellular matrix accumulation in the kidney: implications for diabetic nephropathy. PLOS ONE 8, e77468 (2013).

30. Hajarnis, S. S. et al. Transcription factor hepatocyte nuclear factor-1 $\beta$ (HNF-1 $\beta$ ) regulates microRNA-200 expression through a long noncoding RNA. J. Biol. Chem. 290, 24793-24805 (2015).

31. Mukhopadhyay, D., Houchen, C. W., Kennedy, S., Dieckgraefe, B. K. \& Anant, S. Coupled mRNA stabilization and translational silencing of cyclooxygenase- 2 by a novel RNA binding protein, CUGBP2. Mol. Cell 11, 113-126 (2003).

32. Fiore, R. et al. Mef2-mediated transcription of the miR379-410 cluster regulates activity-dependent dendritogenesis by fine-tuning Pumilio2 protein levels. EMBO J. 28, 697-710 (2009).

33. Lazzaretti, D., Tournier, I. \& Izaurralde, E. The C-terminal domains of human TNRC6A, TNRC6B, and TNRC6C silence bound transcripts independently of Argonaute proteins. RNA 15, 1059-1066 (2009).

34. Novoa, I., Gallego, J., Ferreira, P. G. \& Mendez, R. Mitotic cell-cycle progression is regulated by CPEB1 and CPEB4-dependent translational control. Nat. Cell Biol. 12, 447-456 (2010).

35. de Silanes, I. L., Zhan, M., Lal, A., Yang, X. \& Gorospe, M. Identification of a target RNA motif for RNA-binding protein HuR. Proc. Natl Acad. Sci. USA 101, 2987-2992 (2004).

36. Lan, F. et al. Recognition of unmethylated histone $\mathrm{H} 3$ lysine 4 links $\mathrm{BHC} 80$ to LSD1-mediated gene repression. Nature 448, 718-722 (2007).

37. Hirao, K. et al. EDEM3, a soluble EDEM homolog, enhances glycoprotein endoplasmic reticulum-associated degradation and mannose trimming. J. Biol. Chem. 281, 9650-9658 (2006).

38. Salmena, L., Carracedo, A. \& Pandolfi, P. P. Tenets of PTEN tumor suppression. Cell 133, 403-414 (2008).

39. Fabian, M. R., Sonenberg, N. \& Filipowicz, W. Regulation of mRNA translation and stability by microRNAs. Annu. Rev. Biochem. 79, 351-379 (2010).

40. Wolfgang, C. D., Chen, B. P., Martindale, J. L., Holbrook, N. J. \& Hai, T. gadd153/Chop10, a potential target gene of the transcriptional repressor ATF3. Mol. Cell Biol. 17, 6700-6707 (1997).

41. Yoshida, T. et al. ATF3 protects against renal ischemia-reperfusion injury. J. Am. Soc. Nephrol. 19, 217-224 (2008).

42. Oyadomari, S. \& Mori, M. Roles of CHOP/GADD153 in endoplasmic reticulum stress. Cell Death Differ. 11, 381-389 (2004). 
43. Lan, Y. F. et al. MicroRNA-494 reduces ATF3 expression and promotes AKI. J. Am. Soc. Nephrol. 23, 2012-2023 (2012).

44. Smale, S. T. \& Baltimore, D. The 'initiator' as a transcription control element. Cell 57, 103-113 (1989).

45. Kato, M. et al. MicroRNA-192 in diabetic kidney glomeruli and its function in TGF-beta-induced collagen expression via inhibition of E-box repressors. Proc. Natl Acad. Sci. USA 104, 3432-3437 (2007).

46. Inoki, K. et al. mTORC1 activation in podocytes is a critical step in the development of diabetic nephropathy in mice. J. Clin. Invest. 121, 2181-2196 (2011).

47. Madhusudhan, T. et al. Defective podocyte insulin signalling through p85-XBP1 promotes ATF6-dependent maladaptive ER-stress response in diabetic nephropathy. Nat. Commun. 6, 6496 (2015).

48. $\mathrm{Wu}$, J. et al. Induction of diabetes in aged C57B6 mice results in severe nephropathy: an association with oxidative stress, endoplasmic reticulum stress, and inflammation. Am. J. Pathol. 176, 2163-2176 (2010).

49. Liu, G. et al. Apoptosis induced by endoplasmic reticulum stress involved in diabetic kidney disease. Biochem. Biophys. Res. Commun. 370, 651-656 (2008).

50. Chen, J. et al. ER stress triggers MCP-1 expression through SET7/9-induced histone methylation in the kidneys of $\mathrm{db} / \mathrm{db}$ mice. Am. J. Physiol. Renal Physiol. 306, F916-F925 (2014).

51. Fougeray, S. et al. Metabolic stress promotes renal tubular inflammation by triggering the unfolded protein response. Cell Death Dis. 2, el43 (2011).

52. Yoshida, H., Matsui, T., Yamamoto, A., Okada, T. \& Mori, K. XBP1 mRNA is induced by ATF6 and spliced by IRE1 in response to ER stress to produce a highly active transcription factor. Cell 107, 881-891 (2001).

53. Lee, J. E., Bennett, C. F. \& Cooper, T. A. RNase H-mediated degradation of toxic RNA in myotonic dystrophy type 1. Proc. Natl Acad. Sci. USA 109, 4221-4226 (2012).

54. Michalik, K. M. et al. Long noncoding RNA MALAT1 regulates endothelial cell function and vessel growth. Circ. Res. 114, 1389-1397 (2014).

55. Liang, X. H., Shen, W., Sun, H., Prakash, T. P. \& Crooke, S. T. TCP1 complex proteins interact with phosphorothioate oligonucleotides and can co-localize in oligonucleotide-induced nuclear bodies in mammalian cells. Nucleic Acids Res. 42, 7819-7832 (2014).

56. Susztak, K., Raff, A. C., Schiffer, M. \& Bottinger, E. P. Glucose-induced reactive oxygen species cause apoptosis of podocytes and podocyte depletion at the onset of diabetic nephropathy. Diabetes 55, 225-233 (2006).

57. Weil, E. J. et al. Effect of losartan on prevention and progression of early diabetic nephropathy in American Indians with type 2 diabetes. Diabetes 62, 3224-3231 (2013).

58. Lai, J. Y. et al. MicroRNA-21 in glomerular injury. J. Am. Soc. Nephrol. 26, 805-816 (2015).

59. Miyauchi, M. et al. Hypertrophy and loss of podocytes in diabetic nephropathy. Intern. Med. 48, 1615-1620 (2009).

60. Hodgin, J. B. et al. Glomerular aging and focal global glomerulosclerosis: a podometric perspective. J. Am. Soc. Nephrol. 26, 3162-3178 (2015).

61. Marselli, L. et al. Gene expression profiles of Beta-cell enriched tissue obtained by laser capture microdissection from subjects with type 2 diabetes. PLoS ONE 5, el1499 (2010).

62. Lindenmeyer, M. T. et al. Proteinuria and hyperglycemia induce endoplasmic reticulum stress. J. Am. Soc. Nephrol. 19, 2225-2236 (2008).

63. Borsting, E. et al. Tribbles homolog 3 attenuates mammalian target of rapamycin complex-2 signaling and inflammation in the diabetic kidney. J. Am. Soc. Nephrol. 25, 2067-2078 (2014).

64. Cunard, R. \& Sharma, K. The endoplasmic reticulum stress response and diabetic kidney disease. Am. J. Physiol. Renal Physiol. 300, F1054-F1061 (2011).
65. Wolf, G., Chen, S. \& Ziyadeh, F. N. From the periphery of the glomerular capillary wall toward the center of disease: podocyte injury comes of age in diabetic nephropathy. Diabetes 54, 1626-1634 (2005).

66. Zhu, Y., Casado, M., Vaulont, S. \& Sharma, K. Role of upstream stimulatory factors in regulation of renal transforming growth factor-beta1. Diabetes 54, 1976-1984 (2005).

67. Iglesias-de la Cruz, M. C. et al. Effects of high glucose and TGF-betal on the expression of collagen IV and vascular endothelial growth factor in mouse podocytes. Kidney Int. 62, 901-913 (2002).

68. Ju, W. et al. Tissue transcriptome-driven identification of epidermal growth factor as a chronic kidney disease biomarker. Sci. Transl. Med. 7, 316ra193 (2015).

69. Benjamini, Y. \& Hochberg, Y. Controlling the false discovery rate: a practical and powerful approach to multiple testing. J. R. Stat. Soc. Ser. B (Methodological) 57, 289-300 (1995).

\section{Acknowledgements}

We are grateful to Varun Dang and Katharine Ishida for technical assistance (RT-PCRs), and to members of the Natarajan laboratory for helpful discussions. Supported by grants from the National Institutes of Health (NIH), R01 DK081705, R01 DK058191 and R01 HL106089 (to RN), R01 DK100449 (to MB) and by the Intramural Research Program of the National Institute of Diabetes and Digestive and Kidney Diseases (to RGN). Research reported in this publication included work performed in the following Cores: Pathology, Integrative Genomics, Analytical Pharmacology and DNA/RNA synthesis, supported by the National Cancer Institute of the NIH under award number P30CA33572. EM studies were conducted in the City of Hope EM/AFM Core.

\section{Author contributions}

M.K., M.W., Z.C. and R.N. designed research; M.K., M.W., S.D., K.B., Y.J., H.J.O. and L.L. performed research; M.K., Z.C., M.W. and S.D. analysed the data; J.Y.C.L., Y.W., C.L.O., J.B.H., R.G.N. and M.B. performed research and analysed data with human kidney samples, and edited the manuscript; M.K., Z.C. and R.N., wrote and edited the manuscript. All authors have read and discussed the manuscript.

\section{Additional information}

Supplementary Information accompanies this paper at http://www.nature.com/ naturecommunications

Competing financial interests: The authors declare no competing financial interests.

Reprints and permission information is available online at http://npg.nature.com/ reprintsandpermissions/

How to cite this article: Kato, M. et al. An endoplasmic reticulum stress-regulated lncRNA hosting a microRNA megacluster induces early features of diabetic nephropathy. Nat. Commun. 7:12864 doi: 10.1038/ncomms12864 (2016).

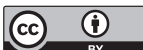

This work is licensed under a Creative Commons Attribution 4.0 International License. The images or other third party material in this article are included in the article's Creative Commons license, unless indicated otherwise in the credit line; if the material is not included under the Creative Commons license, users will need to obtain permission from the license holder to reproduce the material. To view a copy of this license, visit http://creativecommons.org/licenses/by/4.0/

(C) The Author(s) 2016 